\title{
Satellite observations of stratospheric carbonyl fluoride
}

\author{
J. J. Harrison ${ }^{1, *}$, M. P. Chipperfield ${ }^{2}$, A. Dudhia ${ }^{3}$, S. Cai ${ }^{3}$, S. Dhomse ${ }^{2}$, C. D. Boone ${ }^{4}$, and P. F. Bernath ${ }^{1,5}$ \\ ${ }^{1}$ Department of Chemistry, University of York, Heslington, York, YO10 5DD, UK \\ ${ }^{2}$ Institute for Climate and Atmospheric Science, School of Earth and Environment, University of Leeds, Leeds, LS2 9JT, UK \\ ${ }^{3}$ Atmospheric, Oceanic and Planetary Physics, Clarendon Laboratory, University of Oxford, Parks Road, \\ Oxford, OX1 3PU, UK \\ ${ }^{4}$ Department of Chemistry, University of Waterloo, 200 University Avenue West, Ontario N2L 3G1, Canada \\ ${ }^{5}$ Department of Chemistry and Biochemistry, Old Dominion University, Norfolk, Virginia 23529, USA \\ *now at: Space Research Centre, Michael Atiyah Building, Department of Physics and Astronomy, University of Leicester, \\ University Road, Leicester, LE1 7RH, UK
}

Correspondence to: J. J. Harrison (jeremy.harrison@york.ac.uk, jh592@leicester.ac.uk)

Received: 27 May 2014 - Published in Atmos. Chem. Phys. Discuss.: 4 July 2014

Revised: 10 September 2014 - Accepted: 15 September 2014 - Published: 13 November 2014

\begin{abstract}
The vast majority of emissions of fluorinecontaining molecules are anthropogenic in nature, e.g. chlorofluorocarbons (CFCs), hydrochlorofluorocarbons (HCFCs), and hydrofluorocarbons (HFCs). These molecules slowly degrade in the atmosphere, leading to the formation of $\mathrm{HF}, \mathrm{COF}_{2}$, and $\mathrm{COClF}$, which are the main fluorinecontaining species in the stratosphere. Ultimately both $\mathrm{COF}_{2}$ and $\mathrm{COClF}$ further degrade to form HF, an almost permanent reservoir of stratospheric fluorine due to its extreme stability. Carbonyl fluoride $\left(\mathrm{COF}_{2}\right)$ is the secondmost abundant stratospheric "inorganic" fluorine reservoir, with main sources being the atmospheric degradation of CFC-12 $\left(\mathrm{CCl}_{2} \mathrm{~F}_{2}\right)$, HCFC-22 $\left(\mathrm{CHF}_{2} \mathrm{Cl}\right)$, and CFC-113 $\left(\mathrm{CF}_{2} \mathrm{ClCFCl}_{2}\right)$.

This work reports the first global distributions of carbonyl fluoride in the Earth's atmosphere using infrared satellite remote-sensing measurements by the Atmospheric Chemistry Experiment Fourier transform spectrometer (ACEFTS), which has been recording atmospheric spectra since 2004, and the Michelson Interferometer for Passive Atmospheric Sounding (MIPAS) instrument, which recorded thermal emission atmospheric spectra between 2002 and 2012. The observations reveal a high degree of seasonal and latitudinal variability over the course of a year. These have been compared with the output of SLIMCAT, a state-of-the-art three-dimensional chemical transport model. In general the observations agree well with each other, although MIPAS is biased high by as much as $\sim 30 \%$, and compare well with SLIMCAT.
\end{abstract}

Between January 2004 and September $2010 \mathrm{COF}_{2}$ grew most rapidly at altitudes above $\sim 25 \mathrm{~km}$ in the southern latitudes and at altitudes below $\sim 25 \mathrm{~km}$ in the northern latitudes, whereas it declined most rapidly in the tropics. These variations are attributed to changes in stratospheric dynamics over the observation period. The overall $\mathrm{COF}_{2}$ global trend over this period is calculated as $0.85 \pm 0.34$ (MIPAS), $0.30 \pm 0.44$ (ACE), and $0.88 \%$ year $^{-1}$ (SLIMCAT).

\section{Introduction}

Although small quantities of fluorine-containing molecules are emitted into the atmosphere from natural sources, e.g. volcanic and hydrothermal emissions (Gribble, 2002), the vast majority of emissions are anthropogenic in nature, e.g. chlorofluorocarbons (CFCs), hydrochlorofluorocarbons (HCFCs), and hydrofluorocarbons (HFCs). Most fluorine in the troposphere is present in its emitted "organic" form due to these molecules having typical lifetimes of a decade or longer; however photolysis in the stratosphere - which liberates fluorine atoms that react with methane, water, or molecular hydrogen - results in the formation of the "inorganic" product hydrogen fluoride, HF. At the top of the stratosphere ( $\sim 50 \mathrm{~km}$ altitude), $\sim 75 \%$ of the total available fluorine is present as HF (Brown et al., 2014). Due to its extreme stability, HF is an almost permanent reservoir of stratospheric fluorine, meaning the atmospheric concentrations of $F$ and 
FO, necessary for an ozone-destroying catalytic cycle, are very small (Ricaud and Lefevre, 2006). For this reason fluorine does not cause any significant ozone loss. HF is removed from the stratosphere by slow transport to, and rainout in, the troposphere, or by upward transport to the mesosphere, where it is destroyed by photolysis (Duchatelet et al., 2010). The recent stratospheric fluorine inventory for 20042009 (Brown et al., 2014) indicates a year-on-year increase of $\mathrm{HF}$ and total fluorine.

The second-most abundant stratospheric inorganic fluorine reservoir is carbonyl fluoride $\left(\mathrm{COF}_{2}\right)$, largely due to its slow photolysis. Recent studies indicate that its atmospheric abundance is increasing (Duchatelet et al., 2009; Brown et al., 2011). The main sources of $\mathrm{COF}_{2}$ are the atmospheric degradation of CFC-12 $\left(\mathrm{CCl}_{2} \mathrm{~F}_{2}\right)$ and CFC-113 $\left(\mathrm{CF}_{2} \mathrm{ClCFCl}_{2}\right)$, which are both now banned under the Montreal Protocol, and HCFC-22 $\left(\mathrm{CHF}_{2} \mathrm{Cl}\right)$, the most abundant HCFC and classed as a transitional substitute under the Montreal Protocol. Although the amounts of CFC- 12 and CFC-113 in the atmosphere are now slowly decreasing, HCFC-22 is still on the increase. For the two most abundant source molecules, CFC-12 and HCFC-22, the atmospheric degradation proceeds by their initial breakdown into $\mathrm{CF}_{2} \mathrm{Cl}$ (Ricaud and Lefevre, 2006):

$$
\begin{aligned}
& \mathrm{CF}_{2} \mathrm{Cl}_{2}+h v \rightarrow \mathrm{CF}_{2} \mathrm{Cl}+\mathrm{Cl} \\
& \mathrm{CHF}_{2} \mathrm{Cl}+\mathrm{OH} \rightarrow \mathrm{CF}_{2} \mathrm{Cl}+\mathrm{H}_{2} \mathrm{O}
\end{aligned}
$$

followed by

$$
\begin{aligned}
& \mathrm{CF}_{2} \mathrm{Cl}+\mathrm{O}_{2}+M \rightarrow \mathrm{CF}_{2} \mathrm{ClO}_{2}+M \\
& \mathrm{CF}_{2} \mathrm{ClO}_{2}+\mathrm{NO} \rightarrow \mathrm{CF}_{2} \mathrm{ClO}+\mathrm{NO}_{2} \\
& \mathrm{CF}_{2} \mathrm{ClO}+\mathrm{O}_{2} \rightarrow \mathrm{COF}_{2}+\mathrm{ClO}_{2} .
\end{aligned}
$$

For CFC-113 and more minor sources such as HFCs (e.g. HFC-134a, HFC-152a), the reaction scheme is similar.

$\mathrm{COF}_{2}$ volume mixing ratios (VMRs) slowly increase with altitude up to the middle of the stratosphere, above which they decrease as photolysis of $\mathrm{COF}_{2}$ becomes more efficient, leading to the formation of fluorine atoms:

$$
\begin{aligned}
& \mathrm{COF}_{2}+h v \rightarrow \mathrm{FCO}+\mathrm{F} \\
& \mathrm{FCO}+\mathrm{O}_{2}+M \rightarrow \mathrm{FC}(\mathrm{O}) \mathrm{O}_{2}+M \\
& \mathrm{FC}(\mathrm{O}) \mathrm{O}_{2}+\mathrm{NO} \rightarrow \mathrm{FCO}_{2}+\mathrm{NO}_{2} \\
& \mathrm{FCO}_{2}+h v \rightarrow \mathrm{F}+\mathrm{CO}_{2} .
\end{aligned}
$$

As mentioned earlier, these $\mathrm{F}$ atoms react with $\mathrm{CH}_{4}, \mathrm{H}_{2} \mathrm{O}$, or $\mathrm{H}_{2}$ to form HF.

Monitoring $\mathrm{COF}_{2}$ as part of the atmospheric fluorine family is important to close the fluorine budget, particularly as the majority of atmospheric fluorine arises from anthropogenic emissions. Previously, vertical profiles of $\mathrm{COF}_{2}$ in the atmosphere have been determined from measurements taken by the Atmospheric Trace MOlecule Spectroscopy (ATMOS) instrument which flew four times on NASA space shuttles between 1985 and 1994 (Rinsland et al., 1986; Zander et al., 1994). Additionally, there have been several studies into the seasonal variability of $\mathrm{COF}_{2}$ columns above Jungfraujoch using ground-based Fourier transform infrared (FTIR) solar observations (Mélen et al., 1998; Duchatelet et al., 2009). The use of satellite remotesensing techniques allows the measurement of $\mathrm{COF}_{2}$ atmospheric abundances with global coverage, and the investigation more fully of $\mathrm{COF}_{2}$ trends, and seasonal and latitudinal variability. This work presents the first global distributions of $\mathrm{COF}_{2}$ using data from two satellite limb instruments: the Atmospheric Chemistry Experiment Fourier transform spectrometer (ACE-FTS), onboard SCISAT (SCIentific SATellite), which has been recording atmospheric spectra since 2004, and the Michelson Interferometer for Passive Atmospheric Sounding (MIPAS) instrument (Fischer et al., 2008) onboard the ENVIronmental SATellite (Envisat), which recorded thermal emission atmospheric spectra between 2002 and 2012. This work also provides comparisons of these observations with the output of SLIMCAT, a stateof-the-art three-dimensional (3-D) chemical transport model (CTM). Models have not been tested against $\mathrm{COF}_{2}$ observations in detail before; in fact, many standard stratospheric models do not even include fluorine chemistry. Model comparisons with global data sets are essential to test how well $\mathrm{COF}_{2}$ chemistry is understood.

In Sects. 2 and 3 of this paper, full details of the ACE and MIPAS retrieval schemes and associated errors are presented. ACE and MIPAS zonal means and profiles are compared in Sect. 4, with both sets of observations compared with SLIMCAT in Sect. 5. Finally, trends in $\mathrm{COF}_{2}$ VMRs between 2004 and 2010 are calculated and discussed in Sect. 6.

\section{Retrieval of carbonyl fluoride}

\section{$2.1 \quad$ ACE-FTS spectra}

The ACE-FTS instrument, which covers the spectral region 750 to $4400 \mathrm{~cm}^{-1}$ with a maximum optical path difference (MOPD) of $25 \mathrm{~cm}$ and a resolution of $0.02 \mathrm{~cm}^{-1}$ (using the definition of $0.5 / \mathrm{MOPD}$ throughout), uses the sun as a source of infrared radiation to record limb transmission through the Earth's atmosphere during sunrise and sunset ("solar occultation"). Transmittance spectra are obtained by ratioing against exo-atmospheric "high sun" spectra measured each orbit. These spectra, with high signal-to-noise ratios, are recorded through long atmospheric limb paths $(\sim 300 \mathrm{~km}$ effective length), thus providing a low detection threshold for trace species. ACE has an excellent vertical resolution of about $\sim 3 \mathrm{~km}$ (Clerbaux et al., 2005) and can measure up to 30 occultations per day, with each occultation sampling the atmosphere from $150 \mathrm{~km}$ down to the cloud tops (or $5 \mathrm{~km}$ in the absence of clouds). The locations of ACE occultations are dictated by the low Earth circular orbit of SCISAT and the relative position of the sun. Over the course of a year, the ACE-FTS records atmospheric spectra over a large portion of the globe (Bernath et al., 2005). 
The atmospheric pressure and temperature profiles, the tangent heights of the measurements, and the carbonyl fluoride VMRs were taken from the version 3.0 processing of the ACE-FTS data (Boone et al., 2005, 2013). Vertical profiles of trace gases (along with temperature and pressure) are derived from the recorded transmittance spectra via an iterative Levenberg-Marquardt nonlinear least-squares global fit to the selected spectral region(s) for all measurements within the altitude range of interest, according to the equation

$\boldsymbol{x}_{i+1}=\boldsymbol{x}_{i}+\left(\mathbf{K}^{T} \mathbf{S}_{y}^{-1} \mathbf{K}+\lambda \mathbf{I}\right)^{-1} \mathbf{K}^{T} \mathbf{S}_{y}^{-1}\left(\boldsymbol{y}-\mathbf{F}\left(\boldsymbol{x}_{i}, \boldsymbol{b}\right)\right)$.

In Eq. (1), $\boldsymbol{x}$ is the state vector, i.e. the atmospheric quantities to be retrieved; $\boldsymbol{y}$ the vector of measurements (over a range of tangent heights); $\mathbf{S}_{y}$ the measurement error covariance matrix (assumed to be diagonal); $\lambda$ the Levenberg-Marquardt weighting factor; $\mathbf{F}$ the radiative transfer (forward) model; $\boldsymbol{b}$ the forward model parameter vector; $i$ the iteration number; and $\mathbf{K}$ is the Jacobian matrix ( $\equiv \partial \mathbf{F} / \partial \boldsymbol{x}$ ).

The microwindow set and associated altitude ranges are listed in Table 1. The VMRs for molecules with absorption features in the microwindow set (see Table 2) were adjusted simultaneously with the $\mathrm{COF}_{2}$ amount. All spectroscopic line parameters were taken from the HIgh-resolution TRANsmission (HITRAN) database 2004 (Rothman et al., 2005). The v3.0 $\mathrm{COF}_{2}$ retrieval extends from a lower altitude of 12 up to $34 \mathrm{~km}$ at the poles and $45 \mathrm{~km}$ at the Equator, with the upper limit varying with latitude (see Table 1). During the retrieval the state vector is sampled on an altitude grid coinciding with the tangent altitudes of the measurements. The retrieved VMRs are then interpolated onto a uniform $1 \mathrm{~km}$ grid. For ACE spectra recorded at tangent heights that fall within the selected retrieval altitude range, the initial VMRs (which do not vary with season or latitude) for the least-squares fit are taken from the set of VMR profiles established by the ATMOS mission (Irion et al., 2002). The $\mathrm{COF}_{2}$ spectral signal in ACE spectra recorded above the upper-altitude retrieval limit (see Table 1) is generally below the noise level, making it impossible to directly retrieve VMRs at these altitudes. However, the ATMOS profile indicates that the $\mathrm{COF}_{2} \mathrm{VMRs}$ do not effectively drop to 0 until $\sim 55 \mathrm{~km}$. To compensate, the portion of the retrieved VMR profile above the highest analysed ACE measurement is calculated by scaling this ATMOS, or a priori, profile in that altitude region; this scaling factor is determined during the least-squares fitting.

An ACE-FTS transmittance spectrum in the region of one of the microwindows is plotted in the top panel of Fig. 1. This measurement comes from occultation ss11613 (recorded on 9 October 2005 south of Mexico, over the Pacific Ocean) at a tangent height of $28.9 \mathrm{~km}$. The second panel reveals the calculated contribution to the measurement of $\mathrm{COF}_{2}$ based on its retrieved VMR $(\sim 3 \%)$; three spectral features are clearly due to absorption of $\mathrm{COF}_{2}$. The third panel gives the observed-calculated residuals for the retrieval without

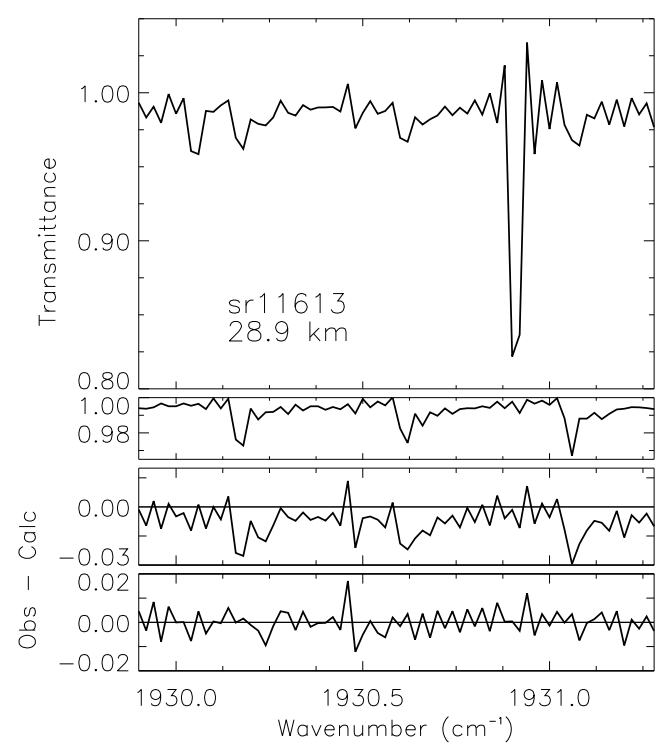

Figure 1. Top panel: an ACE-FTS transmittance spectrum covering the 1929.9-1931.3 $\mathrm{cm}^{-1}$ microwindow for occultation ss 11613 (recorded on 9 October 2005 south of Mexico, over the Pacific Ocean) at a tangent height of $28.9 \mathrm{~km}$. Second panel: the calculated $\mathrm{COF}_{2}$ transmittance contribution to the measurement $(\sim 3 \%)$. Third panel: the observed-calculated residuals for the retrieval without the inclusion of $\mathrm{COF}_{2}$ in the forward model. Bottom panel: the total observed-calculated residuals for the retrieval.

the inclusion of $\mathrm{COF}_{2}$ in the forward model; the shape of these residuals matches well with the calculated $\mathrm{COF}_{2}$ contribution. The bottom panel contains the observed-calculated residuals, indicating the goodness of the fit.

\subsection{MIPAS spectra}

The MIPAS instrument, a Fourier transform spectrometer, measures the thermal limb emission of the Earth's atmosphere in the mid-infrared spectral region, $685-2410 \mathrm{~cm}^{-1}$. Launched in March 2002, the first 2 years of spectra were recorded at an unapodised resolution of $0.025 \mathrm{~cm}^{-1}$ $(\mathrm{MOPD}=20 \mathrm{~cm})$. The nominal scan pattern consisted of 17 tangent points per scan (FR17; FR stands for full resolution) from 6 to $68 \mathrm{~km}$ altitude with a minimum vertical spacing of $3 \mathrm{~km}$. A mechanical degradation of the interferometer's mirror drive led to a cessation in measurements, with a resumption in operations in January 2005 at a reduced resolution of $0.0625 \mathrm{~cm}^{-1}(\mathrm{MOPD}=8 \mathrm{~cm})$. The new nominal scan pattern consisted of 27 tangent points per scan (OR27; OR stands for optimised resolution) over altitude ranges that varied with latitude, from $5-70 \mathrm{~km}$ at the poles to $12-77 \mathrm{~km}$ at the Equator; this variation, which approximately follows the tropopause shape, minimises the number of spectra lost to cloud contamination. The vertical spacing of OR27 scans ranges from $1.5 \mathrm{~km}$ at lower altitudes to 4.5 at higher altitudes. Note that the reduction in scan time associated with 
Table 1. Microwindows for the v3.0 ACE-FTS carbonyl fluoride retrieval.

\begin{tabular}{|c|c|c|c|}
\hline $\begin{array}{l}\text { Centre frequency } \\
\left(\mathrm{cm}^{-1}\right)\end{array}$ & $\begin{array}{l}\text { Microwindow } \\
\text { width }\left(\mathrm{cm}^{-1}\right)\end{array}$ & $\begin{array}{l}\text { Lower altitude } \\
\qquad(\mathrm{km})\end{array}$ & Upper altitude (km) \\
\hline 1234.70 & 1.40 & 12 & $45-11 \sin ^{2}\left(\right.$ latitude $\left.^{\circ}\right)$ \\
\hline 1236.90 & 1.40 & 25 & $45-11 \sin ^{2}\left(\right.$ latitude $\left.^{\circ}\right)$ \\
\hline 1238.00 & 0.80 & 15 & $45-11 \sin ^{2}\left(\right.$ latitude $\left.^{\circ}\right)$ \\
\hline 1239.90 & 1.00 & 15 & $45-11 \sin ^{2}\left(\right.$ latitude $\left.^{\circ}\right)$ \\
\hline 1930.60 & 1.40 & $15-3 \sin ^{2}\left(\right.$ latitude $\left.^{\circ}\right)$ & $45-11 \sin ^{2}\left(\right.$ latitude $\left.^{\circ}\right)$ \\
\hline 1936.48 & 0.65 & 12 & $45-11 \sin ^{2}\left(\right.$ latitude $\left.^{\circ}\right)$ \\
\hline 1938.15 & 1.50 & 30 & $35-6 \sin ^{2}\left(\right.$ latitude $\left.^{\circ}\right)$ \\
\hline 1939.55 & 1.20 & 30 & $35-6 \sin ^{2}\left(\right.$ latitude $\left.^{\circ}\right)$ \\
\hline 1949.40 & 1.20 & 15 & $45-11 \sin ^{2}\left(\right.$ latitude $\left.^{\circ}\right)$ \\
\hline 1950.70 & 0.50 & 12 & $45-11 \sin ^{2}\left(\right.$ latitude $\left.^{\circ}\right)$ \\
\hline 1952.23 & 1.00 & 12 & $45-11 \sin ^{2}$ (latitude $\left.{ }^{\circ}\right)$ \\
\hline $2672.70^{*}$ & 0.60 & 12 & 20 \\
\hline
\end{tabular}

* Included to improve results for interferer HDO.

Table 2. Interferers in the v3.0 ACE-FTS carbonyl fluoride retrieval.

\begin{tabular}{|c|c|c|}
\hline Molecule & $\begin{array}{l}\text { Lower altitude } \\
\text { limit }(\mathrm{km})\end{array}$ & $\begin{array}{l}\text { Upper altitude } \\
\text { limit }(\mathrm{km})\end{array}$ \\
\hline $\mathrm{H}_{2} \mathrm{O}$ & 12 & $45-11 \sin ^{2}$ (latitude $\left.^{\circ}\right)$ \\
\hline $\mathrm{CO}_{2}$ & 12 & $45-11 \sin ^{2}$ (latitude $\left.^{\circ}\right)$ \\
\hline $\mathrm{CH}_{4}$ & 12 & $45-11 \sin ^{2}$ (latitude $\left.^{\circ}\right)$ \\
\hline NO & 12 & $45-11 \sin ^{2}$ (latitude $\left.^{\circ}\right)$ \\
\hline${ }^{13} \mathrm{CH}_{4}$ & 12 & $45-11 \sin ^{2}\left(\right.$ latitude $\left.^{\circ}\right)$ \\
\hline $\mathrm{OC}^{18} \mathrm{O}$ & 12 & $45-11 \sin ^{2}\left(\right.$ latitude $\left.^{\circ}\right)$ \\
\hline $\mathrm{N}_{2} \mathrm{O}$ & 12 & $45-11 \sin ^{2}$ (latitude $\left.^{\circ}\right)$ \\
\hline $\mathrm{N}_{2}^{18} \mathrm{O}$ & 12 & $32-2 \sin ^{2}\left(\right.$ latitude $\left.^{\circ}\right)$ \\
\hline $15^{2} \mathrm{NNO}$ & 12 & $27-2 \sin ^{2}\left(\right.$ latitude $\left.^{\circ}\right)$ \\
\hline HDO & 12 & 24 \\
\hline $\mathrm{CH}_{3} \mathrm{D}$ & 12 & 23 \\
\hline
\end{tabular}

the lower spectral resolution resulted in an increase in the number of tangent points (an additional 10) within the limb scan, thus improving the vertical resolution. MIPAS data are available until April 2012, when communication with the ENVISAT satellite failed.

Retrievals were performed using v1.3 of the Oxford L2 retrieval algorithm MORSE (MIPAS Orbital Retrieval using Sequential Estimation; http://www.atm.ox.ac.uk/MORSE/) with ESA v5 L1B radiance spectra. The equivalent to Eq. (1) in an optimal estimation approach is (e.g. Rodgers, 2000)

$$
\begin{aligned}
\boldsymbol{x}_{i+1} & =\boldsymbol{x}_{i}+\left[(1+\lambda) \mathbf{S}_{\mathrm{a}}^{-1}+\mathbf{K}_{i}^{T} \mathbf{S}_{y}^{-1} \mathbf{K}_{i}^{T}\right]^{-1} \\
& \left\{\mathbf{K}_{i}^{T} \mathbf{S}_{y}^{-1}\left[\boldsymbol{y}-\mathbf{F}\left(\boldsymbol{x}_{i}, \boldsymbol{b}\right)\right]-\mathbf{S}_{\mathrm{a}}^{-1}\left[\boldsymbol{x}_{i}-\boldsymbol{x}_{\mathrm{a}}\right]\right\},
\end{aligned}
$$

where the new terms $\boldsymbol{x}_{\mathrm{a}}$ and $\mathbf{S}_{\mathrm{a}}$ represent the a priori estimate of $\boldsymbol{x}$ and its error covariance, respectively. However, rather than applying the above equation to the full set of measurements $(\boldsymbol{y})$, MORSE uses a sequential estimation approach (Rodgers, 2000) and applies Eq. (2) successively to spectral subsets defined by each microwindow at each tangent height, which varies from scan to scan. For this work, the a priori estimate is taken from IG2 $\mathrm{COF}_{2}$ profiles (Remedios et al., 2007); after each step of the sequential estimation, $\boldsymbol{x}_{\mathrm{a}}$ and $\mathbf{S}_{\mathrm{a}}$ are updated according to the results of the preceding step. The spectral microwindows and associated altitude ranges are listed in Table 3; the retrieval extends from a lower altitude of 7.5 up to $54.0 \mathrm{~km}$, with the retrieved $\mathrm{COF}_{2}$ VMRs interpolated from the tangent altitude grid onto the same $1 \mathrm{~km}$ grid used by ACE. For $\mathrm{COF}_{2}$ retrievals, the MORSE state vector consists of the profile of $\mathrm{COF}_{2}$ plus, for each microwindow (see Table 4), a profile of atmospheric continuum and a radiometric offset (intended to remove any spectrally smooth background variations within each microwindow, e.g. due to aerosols or thin clouds as well as any residual altitude-dependent radiometric offsets). The forward model uses pressure, temperature, and the abundances of major contaminating species $\left(\mathrm{H}_{2} \mathrm{O}\right.$, $\mathrm{O}_{3}, \mathrm{HNO}_{3}, \mathrm{CH}_{4}, \mathrm{~N}_{2} \mathrm{O}$, and $\mathrm{NO}_{2}$ ) retrieved earlier from the same spectra (using MORSE), and IG2 profiles for other minor gases. Spectroscopic data were taken from the MIPAS PF3.2 database (Flaud et al., 2006), with the $\mathrm{COF}_{2}$ data in this compilation coming from the HITRAN 2004 database (Rothman et al., 2005). As with all MORSE VMR retrievals, the initial diagonal elements of $\mathbf{S}_{\mathrm{a}}$ were set to $(100 \%)^{2}$; since MORSE retrieves $\ln (\mathrm{VMR})$ rather than VMR, the $\mathbf{S}_{\mathrm{a}}$ diagonal elements are profile-independent. The off-diagonal elements of $\mathbf{S}_{\mathrm{a}}$ are set assuming a (strong) vertical correlation length of $50 \mathrm{~km}$, which provides regularisation at the expense of vertical resolution. Finally, cloud-contaminated spectra were removed using the cloud index method (Spang et al., 2004) with a threshold value of 1.8. 
Table 3. Microwindows for the MIPAS carbonyl fluoride retrieval.

\begin{tabular}{rrrr}
\hline $\begin{array}{r}\text { Centre Frequency } \\
\left(\mathrm{cm}^{-1}\right)\end{array}$ & $\begin{array}{r}\text { Microwindow } \\
\text { width }\left(\mathrm{cm}^{-1}\right)\end{array}$ & $\begin{array}{r}\text { Lower altitude } \\
(\mathrm{km})\end{array}$ & $\begin{array}{r}\text { Upper altitude } \\
(\mathrm{km})\end{array}$ \\
\hline 773.5000 & 3.0000 & 18.0 & 43.0 \\
1223.9375 & 3.0000 & 10.5 & 54.0 \\
1227.21875 & 2.9375 & 16.5 & 46.0 \\
1231.8750 & 3.0000 & 12.0 & 40.0 \\
1234.7500 & 2.1250 & 7.5 & 19.5 \\
\hline
\end{tabular}

Note that, unlike the ACE-FTS retrievals, MORSE retrieves $\mathrm{COF}_{2}$ at altitudes well above the VMR maximum, even though the information at high altitude is almost entirely from the a priori profiles. Thus, any special treatment to scale the a priori is not required, although, through the vertical correlation, the effect is similar to that explicitly applied for ACE. Additionally, unlike ACE, MORSE uses MIPAS spectra with the Norton-Beer strong apodisation applied; hence $\mathbf{S}_{y}$ is banded rather than diagonal.

Figure 2 provides a plot that illustrates the $\mathrm{COF}_{2}$ spectral feature in one of the MIPAS microwindows. The top panel shows an averaged MIPAS radiance spectrum (in black) interpolated to $20 \mathrm{~km}$ altitude from equatorial measurements taken in March 2010 for the $772-775 \mathrm{~cm}^{-1}$ microwindow; in red is the averaged calculated spectrum based on the averaged retrieved VMRs, but without the inclusion of $\mathrm{COF}_{2}$ in the forward model. The second panel reveals the averaged calculated $\mathrm{COF}_{2}$ contribution to the spectrum. The third panel gives the observed-calculated residuals for the retrieval (in black), again without the calculated $\mathrm{COF}_{2}$ contribution; the shape of these residuals matches well with the calculated $\mathrm{COF}_{2}$ contribution in the second panel. Overlaid in red are the overall observed-calculated residuals, indicating the goodness of the retrieval.

\section{Retrieval errors}

\subsection{Infrared spectroscopy of carbonyl fluoride}

Both ACE-FTS and MIPAS retrievals make use of the $\mathrm{COF}_{2}$ linelist first released as part of the HITRAN 2004 database (and remaining unchanged for the HITRAN 2008 release), with partition data taken from the Total Internal Partition Sums (TIPS) subroutine included in the HITRAN compilation. The retrievals reported here make use of three band systems of $\mathrm{COF}_{2}$; these bands largely correspond to the $v_{1}$ $\left(1943 \mathrm{~cm}^{-1} ; \mathrm{CO}\right.$ stretch $), v_{4}\left(1243 \mathrm{~cm}^{-1} ; \mathrm{CF}_{2}\right.$ antisymmetrical stretch), and $v_{6}\left(774 \mathrm{~cm}^{-1}\right.$; out-of-plane deformation) fundamental modes. In particular, the ACE-FTS retrieval makes use of spectroscopic lines in the $v_{1}$ and $v_{4}$ bands, whereas MIPAS uses $v_{4}$ and $v_{6}$.

Retrieving $\mathrm{COF}_{2}$ VMR profiles from ACE-FTS and MIPAS spectra crucially requires accurate laboratory $\mathrm{COF}_{2}$ spectroscopic measurements. Uncertainty in the laboratory

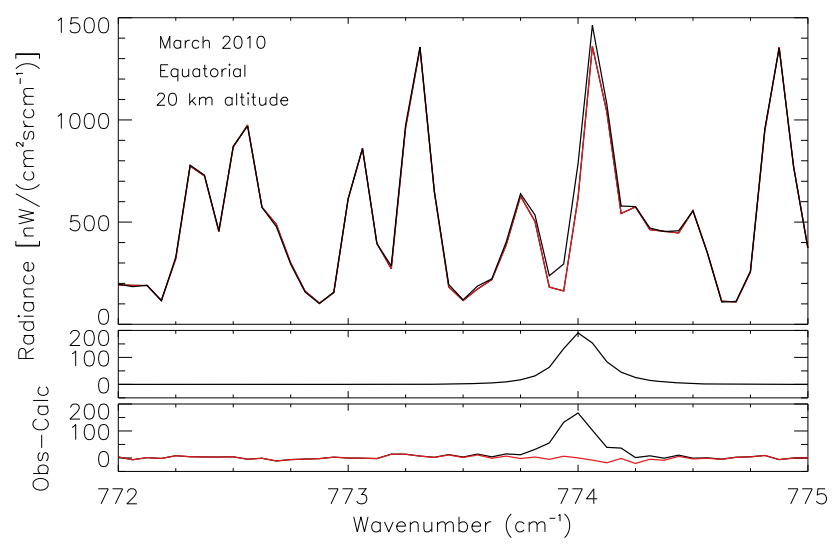

Figure 2. Top panel: an averaged MIPAS radiance spectrum (in black) for equatorial measurements (3547) taken in March 2010 covering the $772-775 \mathrm{~cm}^{-1}$ microwindow and interpolated to $20 \mathrm{~km}$ altitude; in red is the averaged calculated spectrum without the inclusion of $\mathrm{COF}_{2}$ in the forward model. Second panel: the calculated $\mathrm{COF}_{2}$ contribution to the spectrum. Bottom panel: the observed-calculated residuals for the retrieval, with and without $\mathrm{COF}_{2}$ included in the forward model (in red and black, respectively).

Table 4. Interferers in the MIPAS carbonyl fluoride retrieval.

\begin{tabular}{lrr}
\hline Molecule & $\begin{array}{r}\text { Lower altitude } \\
\text { limit }(\mathrm{km})\end{array}$ & $\begin{array}{r}\text { Upper altitude } \\
\text { limit }(\mathrm{km})\end{array}$ \\
\hline $\mathrm{H}_{2} \mathrm{O}$ & 7.5 & 54.0 \\
$\mathrm{CO}_{2}$ & 7.5 & 54.0 \\
$\mathrm{O}_{3}$ & 7.5 & 54.0 \\
$\mathrm{~N}_{2} \mathrm{O}$ & 7.5 & 54.0 \\
$\mathrm{CH}_{4}$ & 7.5 & 54.0 \\
$\mathrm{NO}_{2}$ & 18.0 & 43.0 \\
$\mathrm{HNO}_{3}$ & 10.5 & 54.0 \\
$\mathrm{NH}_{3}$ & 18.0 & 43.0 \\
$\mathrm{HOCl}$ & 7.5 & 54.0 \\
$\mathrm{HCN}$ & 18.0 & 43.0 \\
$\mathrm{H}_{2} \mathrm{O}_{2}$ & 7.5 & 54.0 \\
$\mathrm{CCl}_{4}$ & 18.0 & 43.0 \\
$\mathrm{ClONO}_{2}$ & 18.0 & 43.0 \\
$\mathrm{~N}_{2} \mathrm{O}_{5}$ & 7.5 & 46.0 \\
\hline
\end{tabular}

data can directly contribute to systematic errors in the $\mathrm{COF}_{2}$ retrievals. HITRAN employs error codes in the form of wavenumber errors for the parameters $v$ (line wavenumber) and $\delta_{\text {air }}$ (air-pressure-induced line shift) and percentage errors for $S$ (line intensity), $\gamma_{\text {air }}$ (air-broadened half-width), $\gamma_{\text {self }}$ (self-broadened half-width), and $n_{\text {air }}$ (temperaturedependence exponent for $\gamma_{\text {air }}$ ). Each error code corresponds to an uncertainty range, but with no information as to how the parameters are correlated. In HITRAN the parameter $\delta_{\text {air }}$ for $\mathrm{COF}_{2}$ is assumed to have a value of $0 \mathrm{~cm}^{-1} \mathrm{~atm}^{-1}$. The same values of $\gamma_{\text {air }}\left(0.0845 \mathrm{~cm}^{-1} \mathrm{~atm}^{-1}\right.$ at $\left.296 \mathrm{~K}\right), \gamma_{\text {self }}$ $\left(0.175 \mathrm{~cm}^{-1} \mathrm{~atm}^{-1}\right.$ at $\left.296 \mathrm{~K}\right)$, and $n_{\text {air }}(0.94)$ are used for all 
$\mathrm{COF}_{2}$ spectral lines in HITRAN; according to the error codes these values are averages/estimates. They are taken from the work of May (1992), who determined these average parameters for selected lines in the $v_{4}$ and $v_{6}$ bands from measurements made by a tunable diode-laser spectrometer. For the $v_{1}$ band most of the spectral lines used in the retrievals have stated intensity uncertainties $\geq 20 \%$, for the $v_{4}$ band between 10 and $20 \%$, and for the $\nu_{6}$ band the errors are listed as unreported/unavailable. After performing the MIPAS retrievals, the latest HITRAN 2012 update was released, which revises the $v_{6}$ band and includes several weak hot bands. The listed intensity uncertainties for this band have been revised to between 10 and $20 \%$; spectral simulations indicate only minor intensity differences in the $\nu_{6}$ band $Q$ branch between the two linelists.

As part of the present study, a comparison was made between an $\mathrm{N}_{2}$-broadened (760 Torr) composite spectrum of $\mathrm{COF}_{2}$ (determined from multiple pathlength-concentration burdens) at $278 \mathrm{~K}$ and $0.112 \mathrm{~cm}^{-1}$ resolution, taken from the Pacific Northwest National Laboratory (PNNL) IR database (Sharpe et al., 2004), with a synthetic spectrum calculated using HITRAN $2004 \mathrm{COF}_{2}$ line parameters for the same experimental conditions; the maximum systematic error of the PNNL intensities is $2.5 \%(1 \sigma)$. The comparison reveals that the integrated $v_{1}$ and $v_{4}$ band intensities in the PNNL spectrum are $\sim 15 \%$ higher than HITRAN, whereas the integrated intensity of the very strong $Q$ branch in the $v_{6}$ band of the PNNL spectrum is $\sim 20-25 \%$ higher than HITRAN. Furthermore, the air-broadened half-width in HITRAN for this $Q$ branch appears to be too large at 760 Torr. May (1992) states that the average pressure-broadening coefficients, which are included in HITRAN, could not reproduce the experimental pressure-broadened spectra satisfactorily over the full $Q$ branch region. The author suggests this may be a result of the $J$ (rotational quantum number) dependence of the pressure-broadening coefficients or other effects such as line mixing (Hartmann et al., 2008).

When selecting appropriate ACE microwindows from the $v_{1}$ and $v_{4}$ bands, it was noticed that a number of $\mathrm{COF}_{2}$ lines suffered from systematic bad residuals. Since the $\mathrm{COF}_{2}$ lines occur in clusters, i.e. are not isolated, there is a strong suggestion that line mixing is playing a role; unfortunately there are no available spectroscopic line parameters that describe line mixing for $\mathrm{COF}_{2}$. Although the ACE v3.0 retrieval only employs lines with the best residuals, there could still remain a small contribution to the error from the neglect of line mixing. Lines in the $v_{6} Q$ branch (employed in the MIPAS retrievals) are very tightly packed, so, if line mixing effects are important, errors arising from their neglect will likely be larger for MIPAS retrievals compared with ACE. Unfortunately it is an almost impossible task to quantify these errors without accurate quantitative measurements at low temperatures and pressures. For the purposes of this work it is estimated that retrieval errors arising from $\mathrm{COF}_{2}$ spectroscopy are at most $\sim 15 \%$; however since different bands are used in the respective retrievals, it is likely there will be a relative spectroscopic-induced bias between the two schemes.

\section{$3.2 \quad$ ACE-FTS spectra}

The ACE v2.2 $\mathrm{COF}_{2}$ data product has previously been validated against measurements taken by the JPL MkIV interferometer, a balloon-borne solar occultation FTS (Velazco et al., 2011). Unlike the v3.0 product, the upper-altitude limit for the v2.2 retrieval is fixed at $32 \mathrm{~km}$, with the scaled ACE a priori profile used above $32 \mathrm{~km}$. MkIV and ACE v2.2 profiles from 2004 and 2005 agree well within measurement error, with the relative difference in mean VMRs less than $\sim 10 \%$. However, it must be recognised that both retrievals make use of the same $\mathrm{COF}_{2}$ spectroscopic data, which has an estimated systematic error of at most $\sim 15 \%$ (see Sect. 3.1).

For a single ACE profile, the $1 \sigma$ statistical fitting errors are typically $\sim 10-30 \%$ over most of the altitude range. These errors are random in nature and are largely determined by the measured signal-to-noise ratios of the ACE-FTS spectra, i.e. measurement noise. For averaged profiles, the random errors are small (reduced by a factor of $1 / \sqrt{ } N$, where $N$ is the number of profiles averaged) and the systematic errors dominate.

Spectroscopic sources of systematic error predominantly arise from the $\mathrm{COF}_{2}$ HITRAN linelist $(\sim 15 \%$; see Sect. 3.1), with minor contributions from interfering species that absorb in the microwindow regions. Since the baselines of the ACE-FTS transmittance spectra and the VMRs of the interferers $\left(\mathrm{H}_{2} \mathrm{O}, \mathrm{CO}_{2}, \mathrm{O}_{3}, \mathrm{~N}_{2} \mathrm{O}, \mathrm{CH}_{4}, \mathrm{NO}_{2}, \mathrm{NH}_{3}, \mathrm{HNO}_{3}\right.$, $\mathrm{HOCl}, \mathrm{HCN}, \mathrm{H}_{2} \mathrm{O}_{2}, \mathrm{CCl}_{4}, \mathrm{ClONO}_{2}, \mathrm{~N}_{2} \mathrm{O}_{5}$ ) are fitted simultaneously with the $\mathrm{COF}_{2} \mathrm{VMR}$, it is not a trivial exercise to determine how much they contribute to the overall systematic error of the $\mathrm{COF}_{2}$ retrieval. In this work, the view is taken that the lack of systematic features in the spectral residuals indicates that these contributions are small, at most $1 \%$.

In addition to spectroscopic errors, uncertainties in temperature, pressure, tangent altitude (i.e. pointing), and instrumental line shape (ILS) all contribute to systematic errors in the retrieved $\mathrm{COF}_{2}$ profiles. To estimate the overall systematic error, the retrieval was performed for small subsets of occultations by perturbing each of these quantities $\left(b_{j}\right)$ in turn by its assumed $1 \sigma$ uncertainty $\left(\Delta b_{j}\right)$, while keeping the others unchanged. The fractional retrieval error, $\mu_{j}$, is defined as

$\mu_{j}=\left|\frac{\operatorname{VMR}\left(b_{j}+\Delta b_{j}\right)-\operatorname{VMR}\left(b_{j}\right)}{\operatorname{VMR}\left(b_{j}\right)}\right|$.

Note that, for the ACE-FTS retrievals, pressure, temperature and tangent height are not strictly independent quantities; tangent heights are determined from hydrostatic equilibrium, and so these quantities are strongly correlated. For the purposes of this work, only two of these quantities are altered: temperature is adjusted by $2 \mathrm{~K}$ and tangent height by $150 \mathrm{~m}$ (Harrison and Bernath, 2013). Additionally, ILS uncertainty 
Table 5. Sources of systematic uncertainty in the ACE-FTS v3.0 carbonyl fluoride retrieval.

\begin{tabular}{llr}
\hline Source & Symbol & Fractional value \\
\hline COF$_{2}$ spectroscopy & $\mu_{\text {spec }}$ & 0.15 \\
Spectral interferers & $\mu_{\text {int }}$ & 0.01 \\
Temperature & $\mu_{T}$ & 0.04 \\
Altitude & $\mu_{z}$ & 0.04 \\
ILS & $\mu_{\text {ILS }}$ & 0.01 \\
\hline
\end{tabular}

is induced by adjusting the field of view by $5 \%$ (Harrison and Bernath, 2013). A small subset of occultations was selected for this analysis. The fractional value estimates of the systematic uncertainties, and their symbols, are given in Table 5. Assuming these quantities are uncorrelated, the overall systematic error in the $\mathrm{COF}_{2}$ retrieval can be calculated as

$\mu_{\text {systematic }}^{2}=\mu_{\text {spec }}^{2}+\mu_{\text {int }}^{2}+\mu_{T}^{2}+\mu_{z}^{2}+\mu_{\text {ILS }}^{2}$.

The total systematic error contribution to the ACE-FTS $\mathrm{COF}_{2}$ retrieval is estimated to be $\sim 16 \%$.

As discussed in Sect. 3.1, the $\mathrm{COF}_{2}$ absorption signal in ACE-FTS spectra decreases relative to the noise as the retrieval extends to higher altitude despite the a priori profile indicating that the $\mathrm{COF}_{2} \mathrm{VMRs}$ do not effectively drop to 0 until $\sim 55 \mathrm{~km}$. For this reason an upper-altitude limit (see Table 1) is set; the retrieval is pushed as high in altitude as possible. The portion of the retrieved VMR profile above the highest analysed ACE measurement (i.e. the spectrum at the highest tangent height, just below the upper-altitude limit) is calculated by scaling the a priori profile.

In an ACE retrieval, the calculated spectrum is generated from the sum of contributions from the tangent layer up to $150 \mathrm{~km}$. For the highest analysed measurement, the retrieved VMR in the tangent layer is generated from the piecewise quadratic interpolation scheme (Boone et al., 2005, 2013), while the VMR in every layer above that will come from scaling the a priori profile; the scaling factor largely comes from forcing the calculated spectrum to match as best as possible the measured spectrum for this one measurement. If the shape of the a priori profile above the highest analysed measurement is incorrect, the contribution to the calculated spectrum from that altitude region will be incorrect for the second-highest measurement analysed; the VMRs between the tangent layers of the two highest analysed measurements are adjusted in the retrieval to compensate. Therefore, errors in the a priori VMR profile will introduce systematic errors into the highest altitudes of the retrieved profile.

For the ACE-FTS, the vertical resolution is defined by the sampling unless the separation between measurements is less than the extent of the field of view, in which case the vertical resolution is limited to $\sim 3 \mathrm{~km}$. Although there is some variation in vertical resolution with the beta angle of the measurement, it is often the case that the vertical resolution at high altitudes (above $\sim 40 \mathrm{~km}$ ) is limited by the sampling, while at low altitudes it is limited by the field of view.

\subsection{MIPAS spectra}

The precision, or random error, of the retrieved $\mathrm{COF}_{2}$ VMRs is calculated via the propagation of the instrument noise and the a priori error through the standard optimal estimation retrieval (using the MORSE code). The total retrieval covariance matrix (neglecting systematic errors) is given by (Rodgers, 2000)

$\hat{\mathbf{S}}=\mathbf{S}_{\mathrm{a}}-\mathbf{S}_{\mathrm{a}} \mathbf{K}^{T}\left(\mathbf{K S}_{\mathrm{a}} \mathbf{K}^{T}+\mathbf{S}_{y}\right)^{-1} \mathbf{K S}_{\mathrm{a}}$.

Note that this expression effectively represents a combination of the noise-induced random error and the assumed a priori error covariance (this a priori contribution to the retrieval error is sometimes called "smoothing error"), and that some caution is required if interpolating error profiles to different grids (von Clarmann, 2014). Profile levels with random errors larger than $70 \%$, mostly at the top and bottom of the retrieval range, are discarded from the data set and not used in the analysis. Since the a priori profiles have an assumed error of $100 \%$, this ensures that the retrieved profile levels contain, at worst, $\sim 50 \%$ contribution from the a priori. For a single profile, the noise error is typically $5-15 \%$ between 20 and $40 \mathrm{~km}$, covering the peak of the $\mathrm{COF}_{2}$ VMR profile; over this range the contribution to the retrieved profiles principally comes from the measurements. Outside this range, the errors increase rapidly as the $\mathrm{COF}_{2}$ VMR decreases, and the contribution to the retrieved profiles from the a priori increases.

The total error is computed by propagating a number of independent error sources expressed as spectra through the linearised form of Eq. (2), including both spectral correlations and correlations through the pressure-temperature retrieval. For a single profile, the primary error sources are the measurement noise followed by assumed uncertainties in the $\mathrm{O}_{3}$ (stratosphere) and $\mathrm{N}_{2} \mathrm{O}$ (troposphere) concentrations, which typically contribute $15 \%$ uncertainty in retrieved $\mathrm{COF}_{2}$ values. Spectroscopic errors, including those of interfering species, are treated simply as a single, correlated error source. For $\mathrm{COF}_{2}$ it is assumed that there is an uncertainty of $0.001 \mathrm{~cm}^{-1}$ in line position, $15 \%$ in line strength and $0.1 \mathrm{~cm}^{-1}$ in half-width. Figure 3 shows the single-profile error budget for $\mathrm{COF}_{2}$, with total errors typically $20-30 \%$ between 20 and $40 \mathrm{~km}$. Additionally, the conversion of MIPAS $\mathrm{COF}_{2}$ profiles to absolute altitude for comparison with ACE-FTS profiles relies on the MIPAS pointing information, which may lead to a vertical offset of a few hundred metres relative to ACE.

The sensitivity of the MIPAS $\mathrm{COF}_{2}$ retrieval to the true state can be measured using the averaging kernel matrix (Rodgers, 2000), A: 


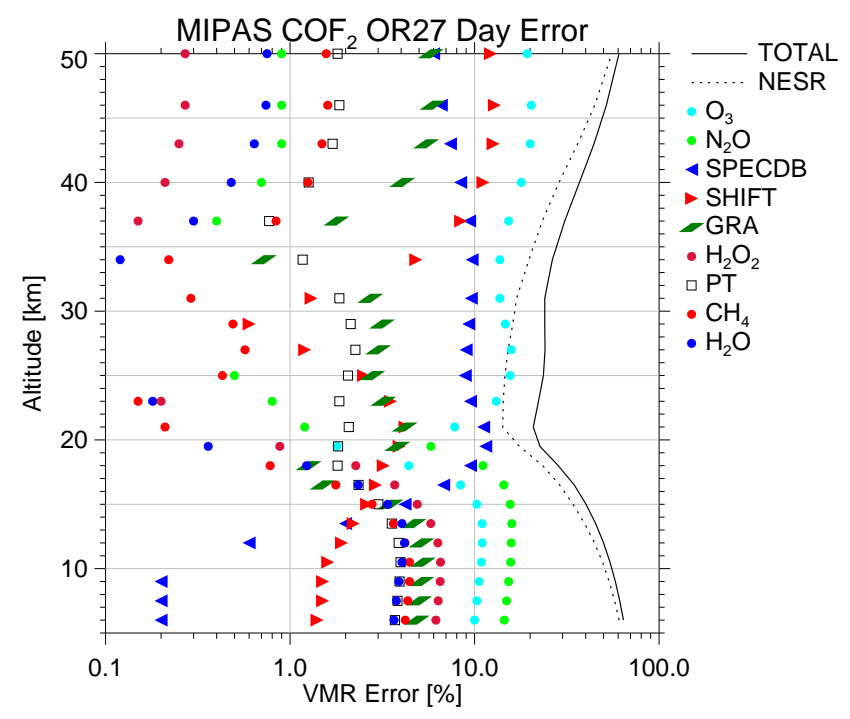

Figure 3. The single-profile total error budget for the MIPAS $\mathrm{COF}_{2}$ retrieval (midlatitude daytime conditions). The total error is computed by propagating a number of independent error sources expressed as spectra through the linearised form of Eq. (2), including both spectral correlations and correlations through the pressuretemperature (PT) retrieval. Note that NESR is the noise equivalent spectral radiance; SHIFT refers to the uncertainty in the spectral calibration $\left( \pm 0.001 \mathrm{~cm}^{-1}\right)$; SPECDB refers to spectroscopic database errors, which are treated simply as a single, correlated error source; and GRA refers to the uncertainty due to an assumed

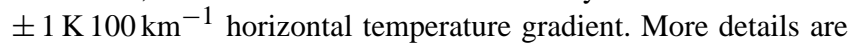
contained in the text. Total errors are typically $20-30 \%$ between 20 and $40 \mathrm{~km}$.

$$
\begin{gathered}
\mathbf{A}=\mathbf{S}_{\mathrm{a}} \mathbf{K}^{T}\left(\mathbf{K} \mathbf{S}_{\mathrm{a}} \mathbf{K}^{T}+\mathbf{S}_{y}\right)^{-1} \mathbf{K}, \\
=\mathbf{I}-\hat{\mathbf{S}} \mathbf{S}_{\mathrm{a}}^{-1}
\end{gathered}
$$

where I is the identity matrix. In general, for a given profile, rows of $\mathbf{A}$ are peaked functions, peaking at the appropriate altitude range for the observation; the width of each function is a measure of the vertical resolution of each $\mathrm{COF}_{2}$ observation.

For the purposes of discussing averaging kernels and vertical resolution of the MIPAS $\mathrm{COF}_{2}$ retrieval, Fig. 4 contains examples of typical retrieved profiles (from 22 December 2011) in cloud-free scenes for north polar winter (NPW), northern midlatitude (MID), Equator (EQU) and south polar summer (SPS) conditions. Averaging kernels (i.e. rows of the averaging kernel matrix) for these four retrievals are presented in Fig. 5. The retrieval altitude of each averaging kernel is indicated by the arrow with matching colour. The MIPAS $\mathrm{COF}_{2}$ retrieval is particularly sensitive in southern polar summer with the combination of high concentrations and high stratospheric temperatures. Figure 6 provides a plot of vertical resolution as a function of altitude for the four retrievals. Vertical resolution is computed as $\mathrm{d} z_{i} / A_{i i}$, where $\mathrm{d} z_{i}$ is the measurement/retrieval grid spacing at profile level $i$

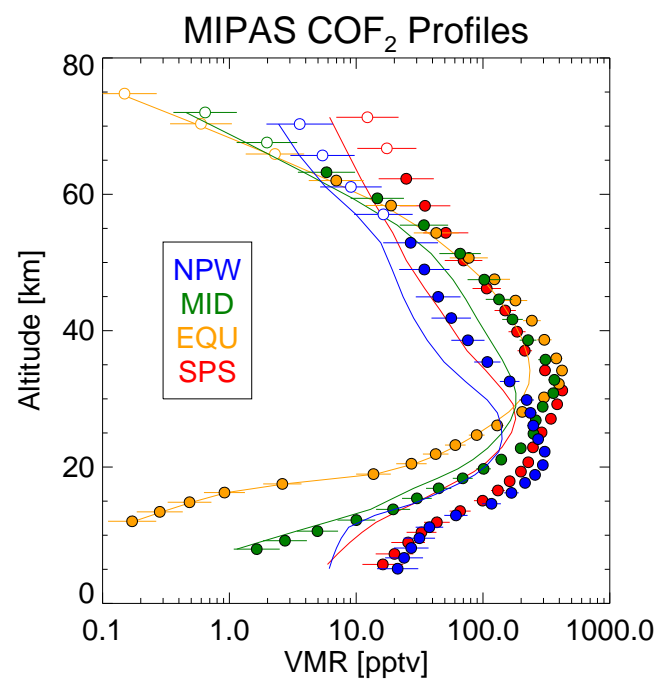

Figure 4. Examples of typical MIPAS retrievals of $\mathrm{COF}_{2}$ profiles in cloud-free scenes for north polar winter (NPW), northern midlatitude (MID), Equator (EQU), and south polar summer (SPS) conditions. Retrieved profiles are shown by circles, with error bars representing the retrieval random error; open symbols are profile levels where this exceeds $70 \%$ of the VMR and so excluded from these analyses. The lines represent the a priori profiles for each retrieval (the a priori error is assumed to be $100 \%$, i.e. a factor of two uncertainty). Profiles are all selected from 22 December 2011, details as follows: NPW Orbit 51319, (80.0 $\left.0^{\circ} \mathrm{N}, 98.8^{\circ} \mathrm{W}\right)$; MID Orbit 51312, $\left(37.6^{\circ} \mathrm{N}, 10.4^{\circ} \mathrm{E}\right)$; EQU Orbit $51312,\left(0.3^{\circ} \mathrm{S}, 96.4^{\circ} \mathrm{W}\right)$; SPS Orbit $51312\left(81.6^{\circ} \mathrm{S}, 44.9^{\circ} \mathrm{E}\right)$.

and $A_{i i}$ is the corresponding diagonal element of the averaging kernel matrix. Figure 6 indicates the vertical resolution of the MIPAS retrievals is $\sim 4-6 \mathrm{~km}$ near the $\mathrm{COF}_{2}$ profile peak, dropping off outside this range.

\section{Global distribution and vertical profiles}

For a detailed comparison between ACE-FTS and MIPAS observations, it was decided to focus on 1 year of measurements between September 2009 and August 2010. Note that, since the differences in vertical resolution between the data sets are not too large, these are not explicitly accounted for in the comparisons. Figure 7 provides a comparison between individual profiles for four near-coincident sets of measurements; these are the four closest sets available over this time period. The locations and times of the eight observations can be found in Table 6 . The plots also include the a priori profiles and calculated SLIMCAT profiles for the location and time of each ACE-FTS observation; these calculations will be discussed in Sect. 5. In Fig. 7, the upper altitudes of the ACE-FTS profiles without error bars correspond to the regions where the a priori profiles are scaled in the retrieval (see Sect. 3.2). Although the pairs of measurements were taken at slightly different locations and times of day, nearcoincident profiles should agree within measurement error, 
Table 6. Near-coincident ACE-FTS and MIPAS measurements.

\begin{tabular}{|c|c|c|c|c|c|c|c|c|}
\hline \multirow{2}{*}{$\begin{array}{l}\text { Date } \\
\text { dd-mm-yyyy }\end{array}$} & \multicolumn{4}{|c|}{ ACE-FTS } & \multicolumn{4}{|c|}{ MIPAS } \\
\hline & Occ & Time (UTC) & Lat & Long & Orbit & Time (UTC) & Lat & Long \\
\hline 03-01-2010 & sr34426 & $13: 22: 21$ & 54.78 & -72.91 & 41018 & $15: 10: 28$ & 54.71 & -72.95 \\
\hline 04-02-2010 & sr34898 & $13: 53: 50$ & 67.27 & -71.25 & 41476 & $15: 01: 10$ & 67.19 & -70.93 \\
\hline $25-05-2010$ & sr36514 & $04: 27: 21$ & 68.86 & -59.05 & 43043 & 02:06:49 & 68.60 & -59.45 \\
\hline $10-07-2010$ & sr37203 & $23: 03: 33$ & -59.27 & -211.3 & 43714 & $23: 56: 31$ & -59.16 & -210.87 \\
\hline
\end{tabular}
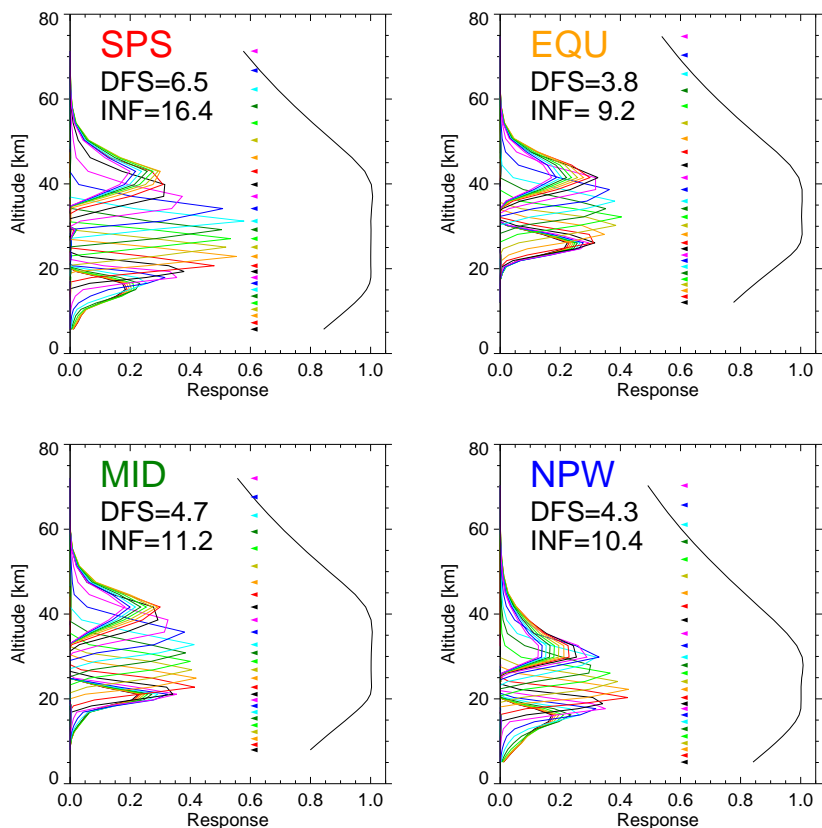

Figure 5. Averaging kernels (i.e. rows of the averaging kernel matrix) of the retrievals shown in Fig. 4. The retrieval altitude of each averaging kernel is indicated by the arrow with matching colour. The solid black line represents the summation of all the elements of each averaging kernel. The figures in each panel refer to "degrees of freedom for signal" (DFS), i.e. the number of independent pieces of information in each profile of 27 levels, which is the trace of the averaging kernel matrix and (INF) Shannon information content (in bits), which includes information from the off-diagonal elements. Of the four regions considered in the plot, the MIPAS $\mathrm{COF}_{2}$ retrieval is most sensitive in southern polar summer with the combination of high concentrations and high stratospheric temperatures.

unless there is significant atmospheric variability. $\mathrm{COF}_{2}$ profiles initially show an increase in VMR with altitude, peaking in the stratosphere and then decreasing with higher altitude; the peak location depends on the latitude and time of year. On the whole, the MIPAS and ACE profiles in Fig. 7 agree well within random error bars. The profile for ACE occultation sr34898 (at high northern latitudes in northern winter) shows a dip near $30 \mathrm{~km}$ due to part of the profile sampling descended $\mathrm{COF}_{2}$-poor upper-stratospheric air within the polar vortex. The near-coincident MIPAS profile does not show

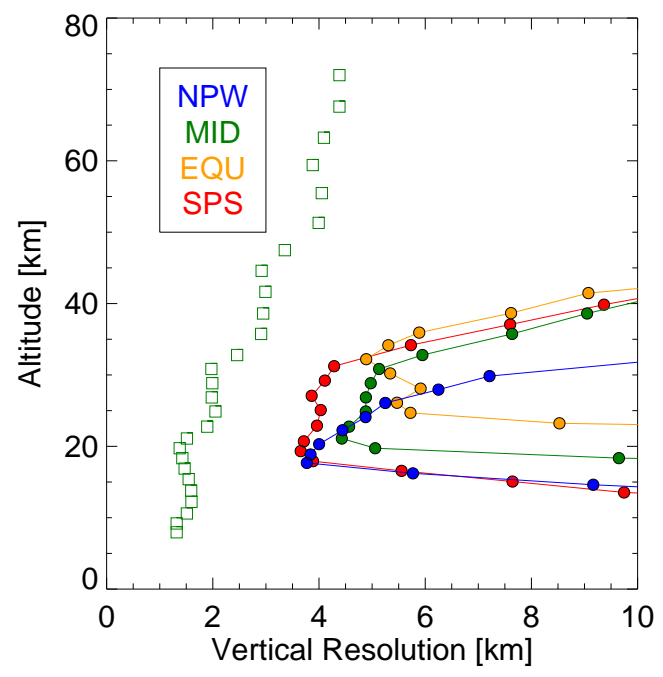

Figure 6. Vertical resolution as a function of altitude of the four retrievals shown in Fig. 4. The open squares show the vertical spacing of the retrieval grid (which is also the measurement tangent height spacing) for the midlatitude profile; for the other profiles the pattern is the same but shifted up or down by a few kilometres. The resolution at each altitude is defined as the ratio of the diagonal of the averaging kernel matrix (Fig. 5) to the grid spacing, which is only meaningful where the averaging kernels have distinct peaks at the tangent point. The MIPAS field of view is approximately $3 \mathrm{~km}$ high, which sets a practical limit on the resolution obtainable at lower altitudes when the limb is oversampled.

such a strong dip, likely due to the poorer vertical resolution of the MIPAS retrieval.

For the preparation of monthly zonal means over the period September 2009 to August 2010, both ACE and MIPAS data sets were filtered to remove those observations deemed "bad". Due to the relatively poor global coverage of ACE observations over this time period, filtering had to be performed carefully; in this case only significant outliers were removed. The MIPAS data set contains substantially more observations over the globe, and, as discussed earlier, profile levels with random errors larger than $70 \%$ of the retrieved VMRs were discarded. For each month, a global spike test was applied to all the remaining data. At each altitude the mean and standard deviation of the ensemble were calculated. Any MIPAS profiles with one or more VMRs outside $5 \sigma$ of the mean VMRs 

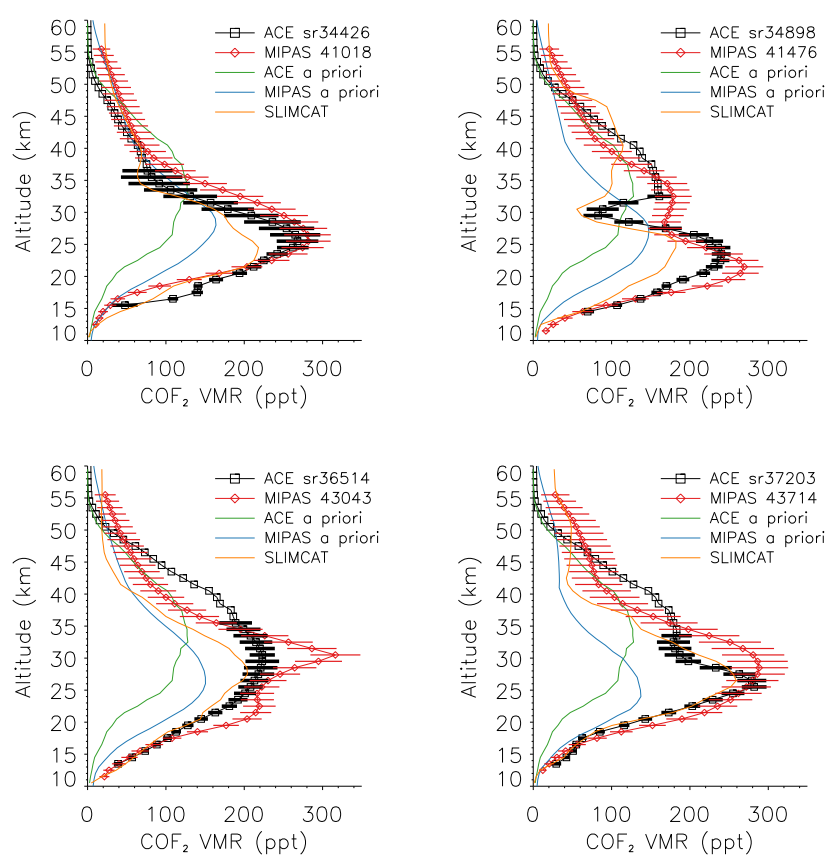

Figure 7. ACE-FTS and MIPAS near-coincident individual profiles taken from the period September 2009 to August 2010. The locations and times of the eight observations can be found in Table 6 . The error bars represent the retrieval random errors. The plots also contain the a priori profiles and calculated SLIMCAT profiles for the location and time of each ACE-FTS observation.

were discarded. This spike test was repeated until all remaining MIPAS profiles were within this $5 \sigma$ range.

MIPAS observations indicate a very minor diurnal variation in $\mathrm{COF}_{2} \mathrm{VMRs}$, well below the measurement error. Therefore, in this work ACE and MIPAS zonal means were produced without any consideration of the local solar time of the individual measurements. Figure 8 provides a direct side-by-side comparison of MIPAS and ACE zonal means for each of the 12 months, revealing the seasonal variation in the $\mathrm{COF}_{2}$ distribution. The plotted VMRs are the averages for each month of all filtered data at each altitude within $5^{\circ}$ latitude bins. The highest $\mathrm{COF}_{2}$ VMRs appear at $\sim 35 \mathrm{~km}$ altitude over the tropics, which receive the highest insolation due to the small solar zenith angle; these peaks are located $\sim 10^{\circ} \mathrm{S}$ for December to April and $\sim 10^{\circ} \mathrm{N}$ for June to October. $\mathrm{COF}_{2}$ has a lifetime of $\sim 3.8$ years (calculated from SLIMCAT; refer to Sect. 5) and is transported polewards by the Brewer-Dobson circulation. As can be seen in the figure, the plots are not symmetric about the Equator. For example, an additional peak at southern high latitudes is most prominent in January/February 2010; this will be further discussed in Sect. 5. The observations in Fig. 8 also demonstrate the presence of a strong Southern Hemisphere (SH) polar vortex in September 2009 and August 2010; the associated low- $\mathrm{COF}_{2}$ VMRs at high southern latitudes are a consequence of the descent of air in the vortex from the upper stratosphere-lower mesosphere, where $\mathrm{COF}_{2}$ VMRs are low. The break-up of the SH polar vortex occurs around November 2009 and begins to form again around June 2010. The Northern Hemisphere $(\mathrm{NH})$ polar vortex is intrinsically weaker and varies considerably from year to year. For the year analysed here the vortex appeared strongest in December 2009 and January 2010. The overall atmospheric distribution of $\mathrm{COF}_{2}$ is determined by a complicated combination of its production, lifetime, and transport. More details on these atmospheric processes will be discussed in Sect. 5, along with a discussion of the SLIMCAT CTM.

Since there are only a maximum of 30 ACE-FTS profiles measured per day, compared to $\sim 1300$ for MIPAS (OR27), the global coverage of the ACE observations between September 2009 and August 2010 is poorer and noisier in appearance. Despite this, the ACE observations agree well with MIPAS, apart from the apparent high bias in the MIPAS VMRs, which will be discussed later in this section. As examples, note the good agreement at mid- to high-latitudes in the SH between regions with high VMRs in December 2009 and March 2010, and low VMRs in August 2010; in the tropical regions, high VMRs peaking north of the Equator in October 2009 and August 2010, and south of the Equator in February 2010; and at mid- to high-latitudes in the NH between regions with high VMRs in September 2009, and low VMRs in February and March 2010.

Since zonal mean plots do not provide an indication of measurement errors, a representative set of individual latitude bins are plotted in Fig. 9 with error bars; all errors are defined as the standard deviations of the bin means. Such plots are useful to inspect biases between data sets. Note that SLIMCAT calculations are also included in this figure; these will be further discussed in Sect. 5. ACE random errors are largest close to the tropics at the highest altitudes of the retrieval (where the black error bars are longest, $\sim 35-$ $45 \mathrm{~km})$. At these altitudes $\mathrm{COF}_{2}$ features in ACE-FTS spectra are weaker, so the relative noise contribution to the retrieved VMRs is larger. The retrieved ACE VMR profiles in this region have a rather flat appearance, whereas the corresponding MIPAS profiles are peaked. The MIPAS VMRs themselves are biased as much as $30 \%$ higher than ACE, although there is overlap between the error bars. This MIPASACE bias is believed to arise predominantly from the large $\mathrm{COF}_{2}$ spectroscopic errors, which make differing contributions to the ACE and MIPAS profiles due to the different microwindows used in the respective retrieval schemes. At the very highest altitudes (above $\sim 50 \mathrm{~km}$ ), the ACE VMRs drop to 0 , and the MIPAS VMRs approach $\sim 50 \mathrm{ppt}$; these differences result from the different a priori profiles used for the two retrieval schemes. A more detailed discussion on this point will be made in Sect. 5. For the August $201025-30^{\circ} \mathrm{S}$ plot in Fig. 9, the increase at the top of the retrieved altitude range (above $\sim 40 \mathrm{~km}$ ) likely results from the approach used to scale the a priori above the highest analysed measurement (refer to Sect. 3.2). Figure 9 also reveals a bias at high 


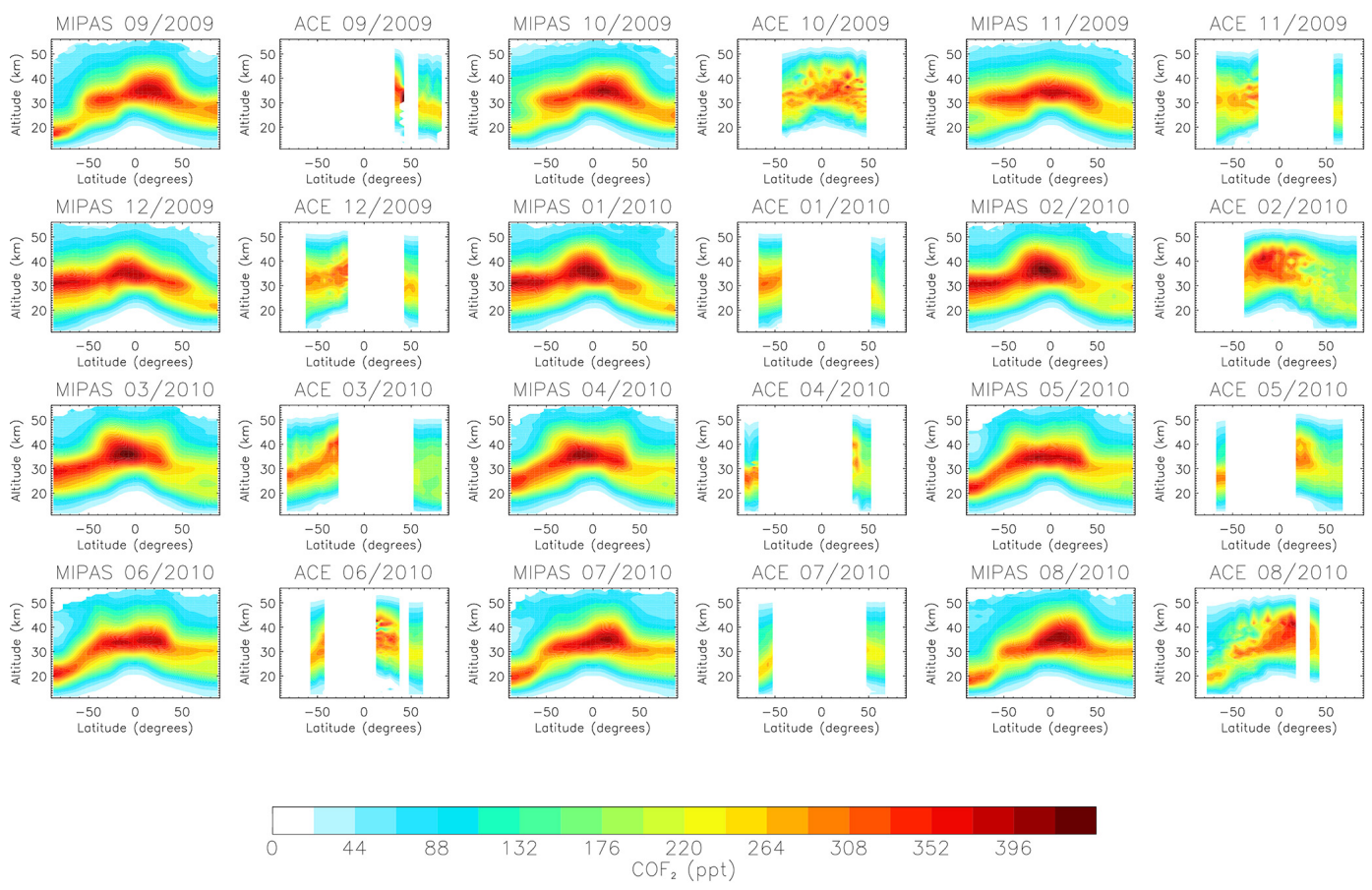

Figure 8. MIPAS and ACE zonal means between September 2009 and August 2010. The plotted VMRs are the averages for each month of all filtered data at each altitude within $5^{\circ}$ latitude bins. Note that the global coverage of the ACE-FTS observations between September 2009

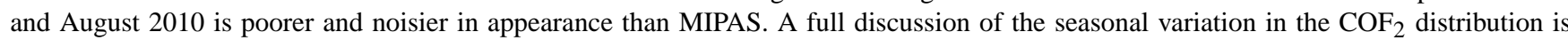
provided in the text.

latitudes in the summer, where the ACE and MIPAS profiles peak just above $30 \mathrm{~km}$. (The summer SH high-latitude peak corresponds to a secondary maximum in the VMR distribution; the origin of this will be discussed in Sect. 5.) As in the tropics, MIPAS VMRs at the peak are $\sim 30 \%$ higher than ACE. Note that, for these particular months, the ACE-FTS was taking many measurements at high latitudes, hence the smaller error bars.

\section{Comparison with SLIMCAT 3-D chemical transport model}

ACE and MIPAS observations have been compared with output from the SLIMCAT off-line 3-D CTM. SLIMCAT calculates the abundances of a number of stratospheric gases from prescribed source-gas surface boundary conditions and a detailed treatment of stratospheric chemistry, including the major species in the $\mathrm{O}_{\mathrm{x}}, \mathrm{NO}_{\mathrm{y}}, \mathrm{HO}_{\mathrm{x}}, \mathrm{Cl}_{\mathrm{y}}$, and $\mathrm{Br}_{\mathrm{y}}$ chemical families (e.g. Chipperfield, 1999; Feng et al., 2007). The model uses winds from meteorological analyses to specify horizontal transport, while vertical motion in the stratosphere is calculated from diagnosed heating rates. This approach gives a realistic stratospheric circulation (Chipperfield, 2006; Monge-Sanz et al., 2007). The troposphere is assumed to be well mixed.
For this study SLIMCAT was integrated from 2000 to 2012 at a horizontal resolution of $5.6^{\circ} \times 5.6^{\circ}$ and $32 \mathrm{lev}-$ els from the surface to $60 \mathrm{~km}$; the levels are not evenly spaced in altitude, but the resolution in the stratosphere is $\sim 1.5-2.0 \mathrm{~km}$. The model uses a $\sigma-\theta$ vertical coordinate (Chipperfield, 2006) and was forced by European Centre for Medium-Range Weather Forecasts (ECMWF) reanalyses (ERA-Interim from 1989 onwards). The volume mixing ratios of source gases at the surface level were specified using data files compiled for the 2010 WMO ozone assessment (WMO/UNEP, 2011). These global mean surface values define the long-term tropospheric source-gas trends in the model.

A previous run of SLIMCAT, used in an investigation of the atmospheric trends of halogen-containing species measured by the ACE-FTS (Brown, et al., 2011), neglected the $\mathrm{COF}_{2}$ contribution from the atmospheric degradation of HFCs. This has now been remedied for the most important HFCs. In total, this run of SLIMCAT calculates $\mathrm{COF}_{2}$ contributions arising from the degradation of CFC-12, CFC-113, CFC-114, CFC-115, HCFC-22, HCFC-142b, HFC-23, HFC134a, HFC-152a, Halon 1211, and Halon 1301. A number of these molecules, e.g. HFC-23, are included even though they make no appreciable contribution to the formation of $\mathrm{COF}_{2}$ compared with the major source gases. Some other HFCs, e.g. HFC-125, which similarly make minimal contribution, are not included in the model. In addition to providing a 

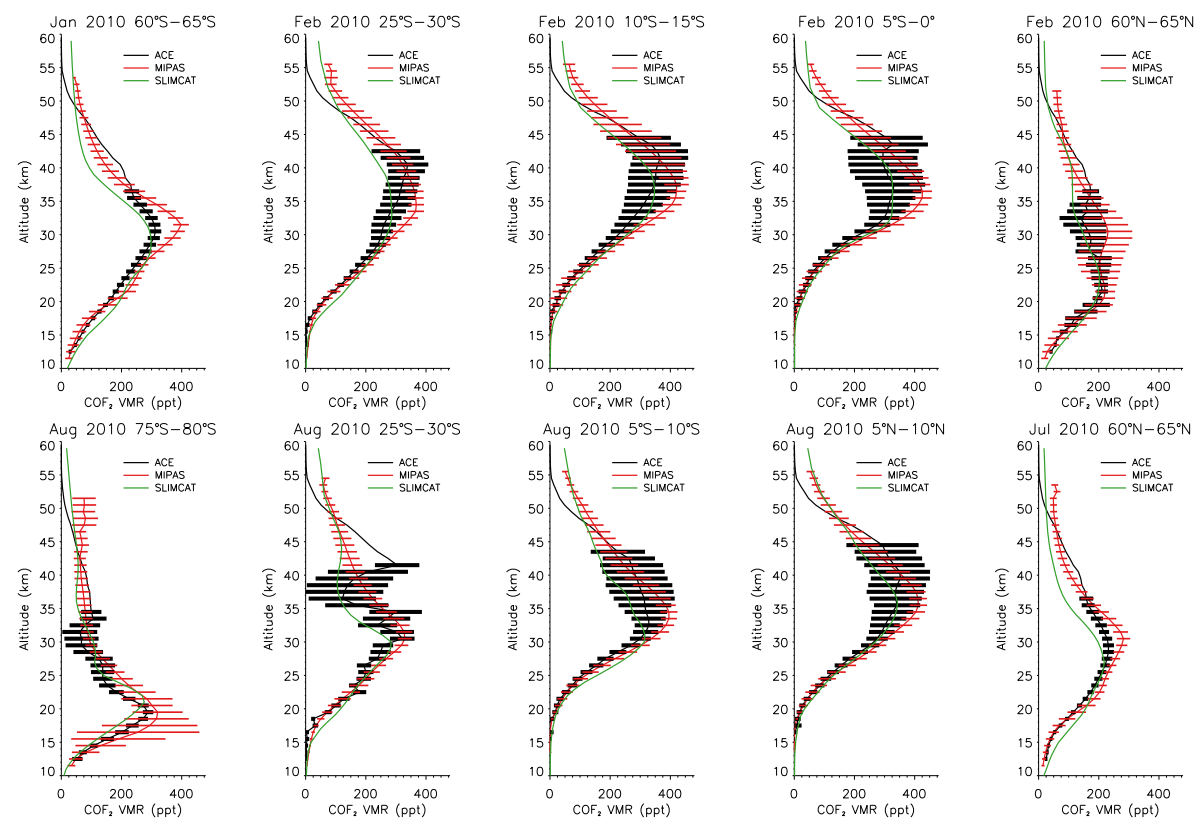

Figure 9. A representative set of MIPAS and ACE individual latitude bins, with errors, taken from Fig. 8. SLIMCAT calculations are also included. A full discussion of the intercomparison is provided in the text.

direct comparison with satellite observations, the new SLIM$\mathrm{CAT}$ calculations have been used to show where $\mathrm{COF}_{2}$ is produced and which source gases have produced it. Most $\mathrm{COF}_{2}$ is produced in the tropics, where solar insolation is highest. Figure 10 provides plots of the loss rates (annual mean zonal mean; pptv day ${ }^{-1}$ ) for the three main source gases which produce $\mathrm{COF}_{2}$. As can be seen, the largest contributing $\mathrm{COF}_{2}$ source at $\sim 30-35 \mathrm{~km}$ is $\mathrm{CFC}-12$, followed by CFC-113 (approximately a factor of 10 smaller). HCFC-22 is the second-largest contributing source gas overall; however its contribution peaks low in the troposphere (not relevant for stratospheric $\left.\mathrm{COF}_{2}\right)$ and higher up in the stratosphere $(\sim 40$ $45 \mathrm{~km}$ ). CFC-12 and CFC-113 are removed mainly by photolysis $\sim 20-40 \mathrm{~km}$; above this altitude range the abundances of CFC-12 and CFC-113 tend to 0 so that they make only a small contribution to the formation of $\mathrm{COF}_{2}$. On the other hand, HCFC-22 is mainly removed from the atmosphere by reaction with $\mathrm{OH}$. Since this reaction is slower, HCFC-22 persists higher into the stratosphere than CFC-12 and CFC113 and can therefore lead to $\mathrm{COF}_{2}$ production in the upper stratosphere and lower mesosphere. Individual contributions from molecules other than these three are typically a small fraction of $1 \%$. In the altitude region below the maximum $\mathrm{COF}_{2} \mathrm{VMRs}$ at all locations there is net production of $\mathrm{COF}_{2}$, while at higher altitudes there is net loss. The primary loss of $\mathrm{COF}_{2}$ in the atmosphere occurs via photolysis, with an additional secondary loss mechanism through reaction with $\mathrm{O}\left({ }^{1} \mathrm{D}\right)$; SLIMCAT calculates the relative contributions as 90 and $10 \%$, respectively. Figure 10 also contains a plot of the $\mathrm{COF}_{2}$ annual mean zonal total loss rate.
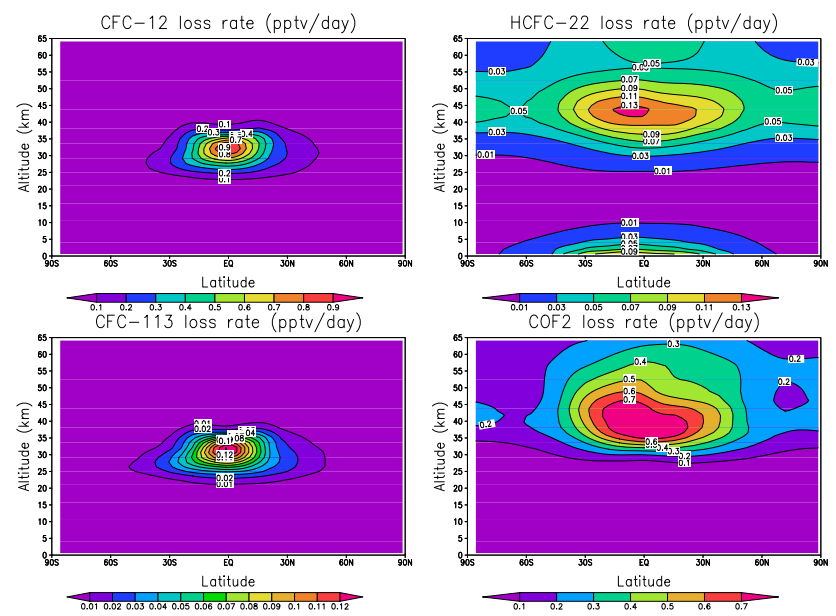

Figure 10. Average loss rates (annual mean zonal mean; pptv day ${ }^{-1}$ ) calculated by SLIMCAT for $\mathrm{COF}_{2}$ and its three main source gases, CFC-12, HCFC-22, and CFC-113. Full details of the loss mechanisms are provided in the text.

SLIMCAT has also been used to estimate the atmospheric lifetime of $\mathrm{COF}_{2}$ by simply dividing the total modelled atmospheric burden by the total calculated atmospheric loss rate. The total calculated mean atmospheric lifetime is $\sim 3.8$ years. This lifetime varies slightly between the hemispheres, 3.76 years in the south and 3.82 years in the north. In the lower stratosphere $\mathrm{COF}_{2}$ can be regarded as a long-lived tracer (local lifetime of many years). Therefore, its tracer isopleths follow the typical tropopause-following contours of 
any long-lived tracer. In this sense, $\mathrm{COF}_{2}$ is analogous to $\mathrm{NO}_{\mathrm{y}}$ which is produced from $\mathrm{N}_{2} \mathrm{O}$. It has been checked as part of this work that a correlation plot of $\mathrm{COF}_{2}$ with its major source, CFC-12, is compact in the lower stratosphere, at altitudes below the region of $\mathrm{COF}_{2}$ maxima (Plumb and Ko, 1992).

As discussed in Sect. 4, Fig. 7 contains a comparison between individual ACE-FTS and MIPAS profiles for the measurements specified in Table 6. This figure also contains SLIMCAT profiles calculated for the location and time of each ACE-FTS observation. In comparison with the retrieved portion of the ACE profiles (marked by black error bars), the calculated SLIMCAT VMRs are generally slightly lower; the agreement with MIPAS is worse, but it must be acknowledged that the two sets of measurements are not strictly coincident. Additionally, SLIMCAT captures the VMR "dip" observed for ACE occultation sr34898 (at $67.27^{\circ} \mathrm{N}$ on the vortex edge, 4 February 2010) near $30 \mathrm{~km}$ altitude, confirming that this profile samples air from the polar vortex. This explanation is supported by the corresponding ACE HF profile, which shows an enhancement near $30 \mathrm{~km}$ due to the sampling of descended HF-rich upper-stratospheric air from the polar vortex.

Figure 11 provides a comparison between SLIMCAT and ACE zonal means. In order to increase the latitude coverage for the comparison and reduce the noise over some of the latitude bands, the plotted ACE data are averages of the data in Fig. 8 (September 2009 to August 2010) with data from the previous year; on the scale of the figure there is no significant variation in the seasonal pattern as measured by the ACE-FTS. Figure 11 reveals that the model agrees well with the ACE observations and reproduces very well the significant seasonal variation, although SLIMCAT produces slightly lower VMRs and the ACE measurements still suffer from measurement noise. Comparing the SLIMCAT zonal means (in Fig. 11) with those for MIPAS (in Fig. 8) again demonstrates the good agreement in seasonal variation, but the MIPAS VMRs have a noticeably high bias compared with the model.

Figure 9 shows a representative set of SLIMCAT profiles in $5^{\circ}$ latitude bins from the September 2009 to $\mathrm{Au}$ gust 2010 time period, along with averaged ACE and MIPAS profiles. These demonstrate a very good agreement between the SLIMCAT calculations and ACE observations, although above $\sim 35 \mathrm{~km}$ this agreement is somewhat worse, particularly the upper parts of the ACE profiles (without error bars) which are derived from the scaled a priori profile and susceptible to systematic errors (see Sect. 3.2). Whereas the ACE VMRs drop to 0 at $\sim 55 \mathrm{~km}$, the SLIMCAT VMRs do not reach 0 even near the model top level around $60 \mathrm{~km}$ due to the calculated ongoing production of $\mathrm{COF}_{2}$ from HCFC22 (see Fig. 10). MIPAS VMRs similarly do not drop to 0 , principally because the a priori profiles make a larger contribution to the retrieved VMRs at these altitudes. Unfortunately, neither ACE nor MIPAS measurements are able to validate the SLIMCAT model HCFC-22/COF 2 VMRs near $55-60 \mathrm{~km}$.

In autumn when solar heating of the relevant polar region comes to an end, a stratospheric polar vortex begins to form. This is a large-scale region of air contained within a strong westerly jet stream that encircles the polar region. Reaching maximum strength in the middle of winter, the polar vortex decays as sunlight returns to the polar region in the spring. Polar vortices, which extend from the tropopause up into the mesosphere, are quasi-containment vessels for air at cold temperatures and low-ozone content. They play a critical role in polar ozone depletion, more so in the Antarctic, where the vortex is larger, stronger, and longer-lived than in the Arctic. The SLIMCAT September 2009 (09/2009) plot in Fig. 11 demonstrates the presence of a strong SH polar vortex by the low- $\mathrm{COF}_{2}$ VMRs at high southern latitudes; as mentioned earlier this is a consequence of the descent of upper-stratospheric air where $\mathrm{COF}_{2} \mathrm{VMRs}$ are very low. The break-up of the SH polar vortex as simulated by SLIMCAT occurs around November 2009 (11/2009) and begins to form again around June $2010(06 / 2010)$. On the other hand, the descent of upper-stratospheric air corresponding to the onset of the $\mathrm{NH}$ polar vortex is less obvious due to the intrinsically lower $\mathrm{COF}_{2}$ VMRs in the NH summer; SLIMCAT observations suggest the northern polar vortex is present from December 2009 to January 2010.

Although some of the $\mathrm{COF}_{2}$ present at mid- and high latitudes can be attributed to transport of $\mathrm{COF}_{2}$-rich tropical air via the Brewer-Dobson circulation (a slow upwelling of stratospheric air in the tropics, followed by poleward drift through the midlatitudes, and descent in the mid- and high latitudes), this cannot account for the secondary maximum in $\operatorname{VMR}(\sim 31 \mathrm{~km})$ present in the SH polar region for which an atmospheric chemistry explanation is needed. Diagnosis of the model rates shows that, in summer, photochemical production of $\mathrm{COF}_{2}$ extends to the pole in the middle stratosphere (i.e. in polar day). Further diagnosis of the first-order loss rates of the main $\mathrm{COF}_{2}$ precursors shows that photolysis and reactions with $\mathrm{O}\left({ }^{1} \mathrm{D}\right)$ are symmetrical between the hemispheres. The only precursor loss reaction which shows significant hemispheric asymmetry is the temperature-dependent reaction of $\mathrm{CHF}_{2} \mathrm{Cl}(\mathrm{HCFC}-22)+\mathrm{OH}$. As the $\mathrm{SH}$ polar summer mid-stratosphere is around $10 \mathrm{~K}$ warmer than the corresponding location in the $\mathrm{NH}$, this reaction provides a stronger source of $\mathrm{COF}_{2}$ in $\mathrm{SH}$ summer compared to the Arctic and contributes to this secondary maximum. Indeed, in a model sensitivity run where the production of $\mathrm{COF}_{2}$ from HCFC22 was switched off, this secondary SH summer peak disappeared. While the first-order loss rates of the $\mathrm{COF}_{2}$ sourcegas precursors are generally symmetrical between the hemispheres, this is not true for the source gases themselves. Differences in the meridional Brewer-Dobson circulation, with stronger mixing to the pole in the north and stronger descent in the south, lead to differences in the distribution of $\mathrm{COF}_{2}$ precursors. This leads to differences in $\mathrm{COF}_{2}$ production, 


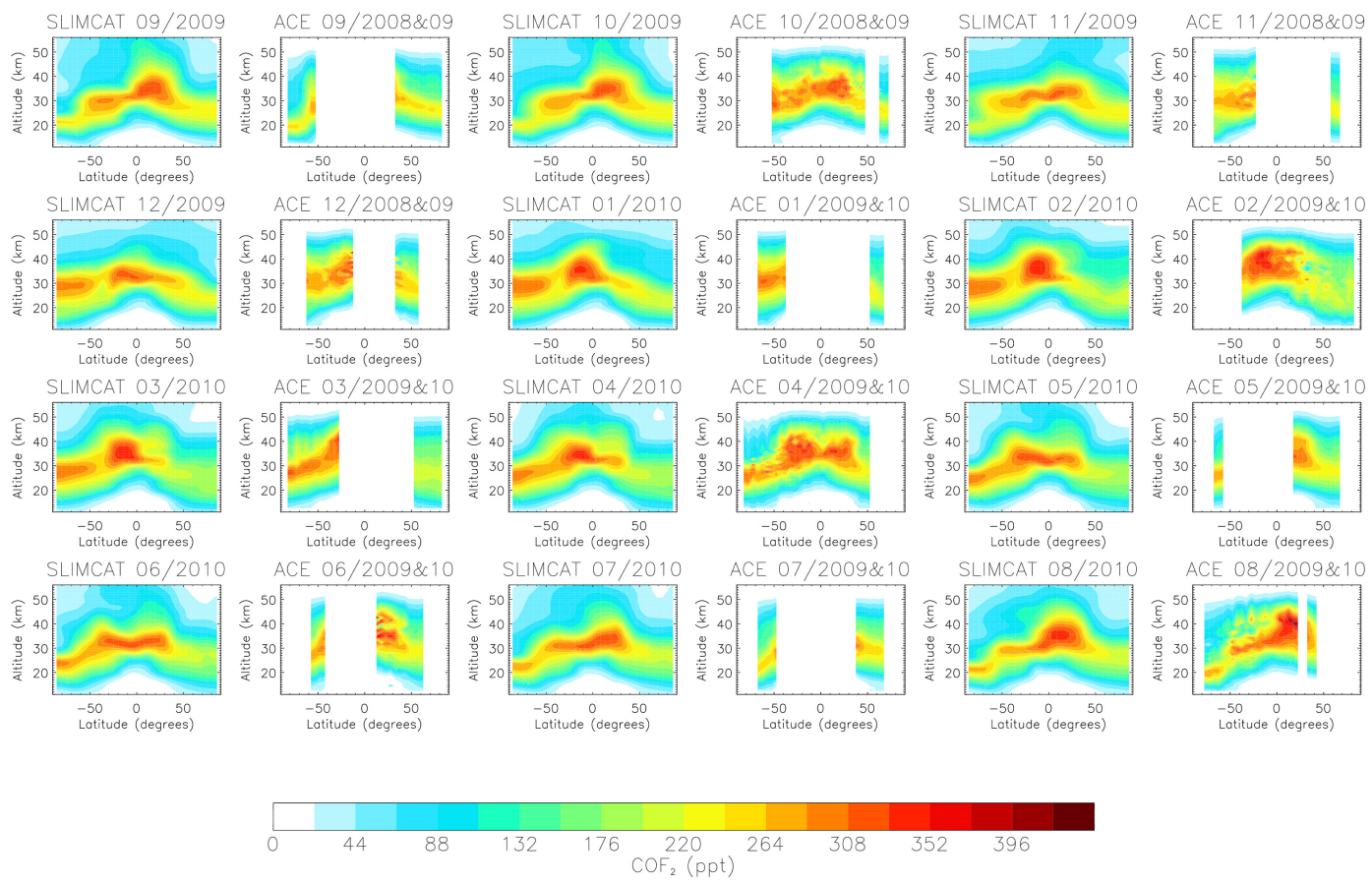

Figure 11. A comparison between monthly SLIMCAT and ACE zonal means (September 2009 to August 2010). In order to reduce the noise and increase the latitude coverage for the comparison, the plotted ACE data have been extended to the previous year. A full discussion of the seasonal variation in the $\mathrm{COF}_{2}$ distribution is provided in the text.

resulting in the observed and modelled hemispheric asymmetry in $\mathrm{COF}_{2}$ at middle latitudes.

\section{Trends}

As mentioned in the Introduction, there is evidence that the atmospheric abundance of $\mathrm{COF}_{2}$ is increasing with time (Duchatelet et al., 2009; Brown et al., 2011). Although the atmospheric abundances of $\mathrm{COF}_{2}$ source gases such as $\mathrm{CFC}$ 12 and CFC-113 are currently decreasing, HCFC-22 and the minor HFC contributors are still on the increase. Figure 1-1 of the 2010 WMO ozone assessment (WMO/UNEP, 2011) shows the trends in mean global surface mixing ratios for these two species during the 1990-2009 time period. The CFC-12 growth rate is observed to reduce slowly from 1990, plateauing around 2003-2004, after which it becomes negative; i.e. there is an overall loss of CFC-12. In comparison, the growth rate of HCFC-22 has been relatively constant since 1990, with a slight increase in growth rate occurring around 2007.

A number of previous studies have quantified the trend in atmospheric $\mathrm{COF}_{2}$ over time. For the Jungfraujoch 1985 to 1995 time series $\left(46.5^{\circ} \mathrm{N}\right.$ latitude, $8.0^{\circ} \mathrm{E}$ longitude), a period when CFC-12 was still increasing in the atmosphere, an average $\mathrm{COF}_{2}$ linear trend of $4.0 \pm 0.5 \%$ year $^{-1}$ was derived (Mélen et al., 1998). $\mathrm{COF}_{2}$ trends from more recent studies are considerably lower, largely due to the phase out of its principal source gas, CFC-12. A trend of $0.8 \pm 0.4 \%$ year $^{-1}$ has recently been derived from ACE data for 2004 to 2010 (Brown et al., 2011). Since the majority of halogenated source gases reach the stratosphere by upwelling through the tropical tropopause region, the $\mathrm{ACE} \mathrm{COF}_{2}$ trend was determined by averaging measurements in the latitude band $30^{\circ} \mathrm{S}$ to $30^{\circ} \mathrm{N}$ between 30 and $40 \mathrm{~km}$ altitude; effectively the seasonal variation in $\mathrm{COF}_{2}$ was averaged out. For the Jungfraujoch 2000 to 2007 time series, a linear trend of $0.4 \pm 0.2 \%$ year $^{-1}$ was derived (Duchatelet et al., 2009). The observed $\mathrm{COF}_{2}$ seasonal variation, which was removed using a cosine function, had maxima towards the end of February (winter) and minima in late summer, when photodissociation processes are at their maximum. In contrast, trends calculated from older SLIMCAT runs for Brown et al. (2011) and Duchatelet et al. (2009) are $-1.3 \pm 0.4$ and $-0.5 \pm 0.2 \%$ year $^{-1}$, respectively. For the latter of these, it was noted that the SLIMCAT time series suffered from several discontinuities in the operational ECMWF meteorological data, for which the vertical resolution had been changed several times; this resulted in a decrease in the SLIMCAT $\mathrm{COF}_{2}$ columns between 2002 and 2006. For the present work, this is no longer a problem because ERA-Interim reanalyses, which use a consistent version of the ECMWF model, are now used by SLIMCAT (e.g. Dhomse et al., 2011).

In this section, ACE and MIPAS time series are derived as a function of altitude and latitude. As discussed previously, 

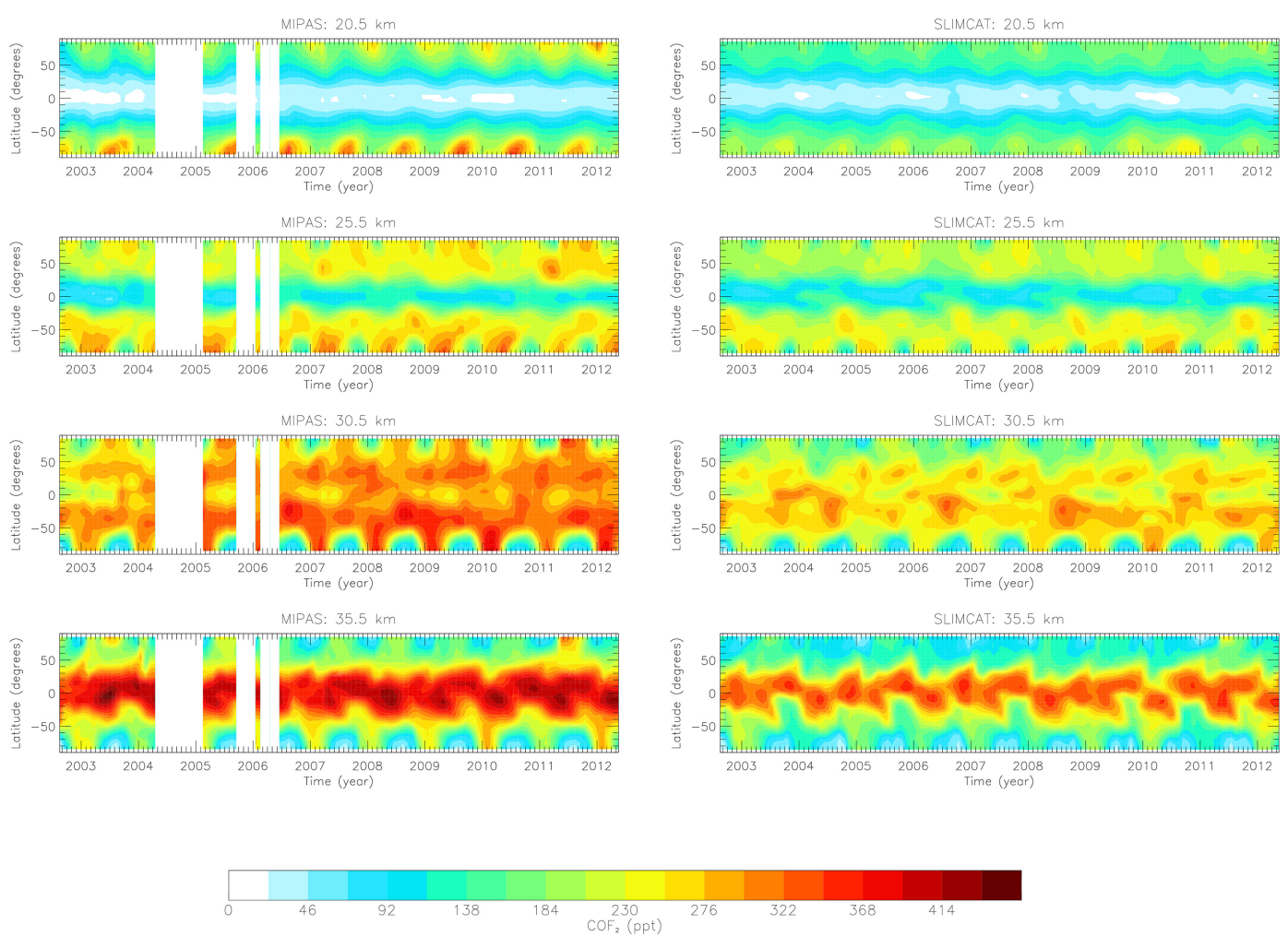

Figure 12. The MIPAS and SLIMCAT COF 2 time series between July 2002 and April 2012 for all latitudes at selected altitudes.

e.g. in Harrison and Bernath (2013), ACE latitude coverage is uneven. For data between January 2004 and September 2010 (the last month for which ACE v3.0 data is usable due to problems with the pressure/temperature a priori), the $1810^{\circ}$ latitude bins used for the ACE time series contain, from southernmost to northernmost, 1000, 1323, 5265, 1776, 796, $608,482,420,390,394,339,413,650,1062,2012,4828$, 1875 , and 1315 occultations; i.e. over three-quarters of the measurements lie in latitude bins poleward of $50^{\circ} \mathrm{S} / \mathrm{N}$. On the other hand, MIPAS data coverage over the globe is more even and extensive, apart from some periods during 20042006 when nominal mode measurements were not made.

Figure 12 illustrates the MIPAS and SLIMCAT time series for $\mathrm{COF}_{2}$ between July 2002 and April 2012 for all latitudes at selected altitudes; both data sets were binned in $10^{\circ}$ latitude bands. (Due to the sparse nature of the ACEFTS measurements, such a plot has not been provided for the ACE data set.) An annual cycle is readily observed, and as expected its phase is opposite in each hemisphere. The amplitude of this cycle is largest near the poles; note that the maxima in the plot at $20.5 \mathrm{~km}$ altitude correspond to the descent of $\mathrm{COF}_{2}$ in winter polar vortices. Close inspection of Fig. 12, particularly the plots above $30 \mathrm{~km}$, also reveals the presence of the quasi-biennial oscillation (QBO) signal, which is strongest in the tropics. Overall, there is good agreement between the MIPAS and SLIMCAT plots in terms of the overall latitude-altitude pattern; however, as noted before, the MIPAS VMRs are biased high - for example, maxima over the tropics as much as $\sim 25 \%$ and maxima near the poles as much as $\sim 50 \%$.

Figure 13 provides the time series for five altitude-latitude bin combinations of ACE, MIPAS, and SLIMCAT data; for ease of viewing, this plot does not include errors. In all plots, the main features in the time series agree well. Note the observed QBO signal for all three data sets, which is stronger in the two tropical plots and weaker in the high-northernlatitude plot. In the top two plots of Fig. 13 MIPAS is biased high, although less so at $20.5 \mathrm{~km}$. As established previously (refer to Fig. 9), this is a feature of the MIPAS data set at the high southern latitudes. The agreement between ACE and SLIMCAT is somewhat better, agreeing within the errors of the ACE data, although less so at high southern latitudes.

$\mathrm{COF}_{2}$ trends at each altitude for all 18 latitude bins have been calculated from monthly percentage anomalies in $\mathrm{COF}_{2}$ zonal means, $\mathrm{C}^{z, \theta}(n)$, defined as

$$
\mathrm{C}^{z, \theta}(n)=100 \frac{\mathrm{VMR}^{z, \theta}(n)-\sum_{m=1}^{12} \delta_{n m}{\overline{\mathrm{VMR}^{z}}}^{z, \theta}(m)}{\sum_{m=1}^{12} \delta_{n m} \overline{\mathrm{VMR}}^{z, \theta}(m)},
$$

where $n$ is a running index from month 0 to 80 (January 2004 to September 2010); $\mathrm{VMR}^{z, \theta}(n)$ is the corresponding mixing ratio at altitude $z$ and latitude $\theta ; \overline{\mathrm{VMR}}^{z, \theta}(m)$ is the average 

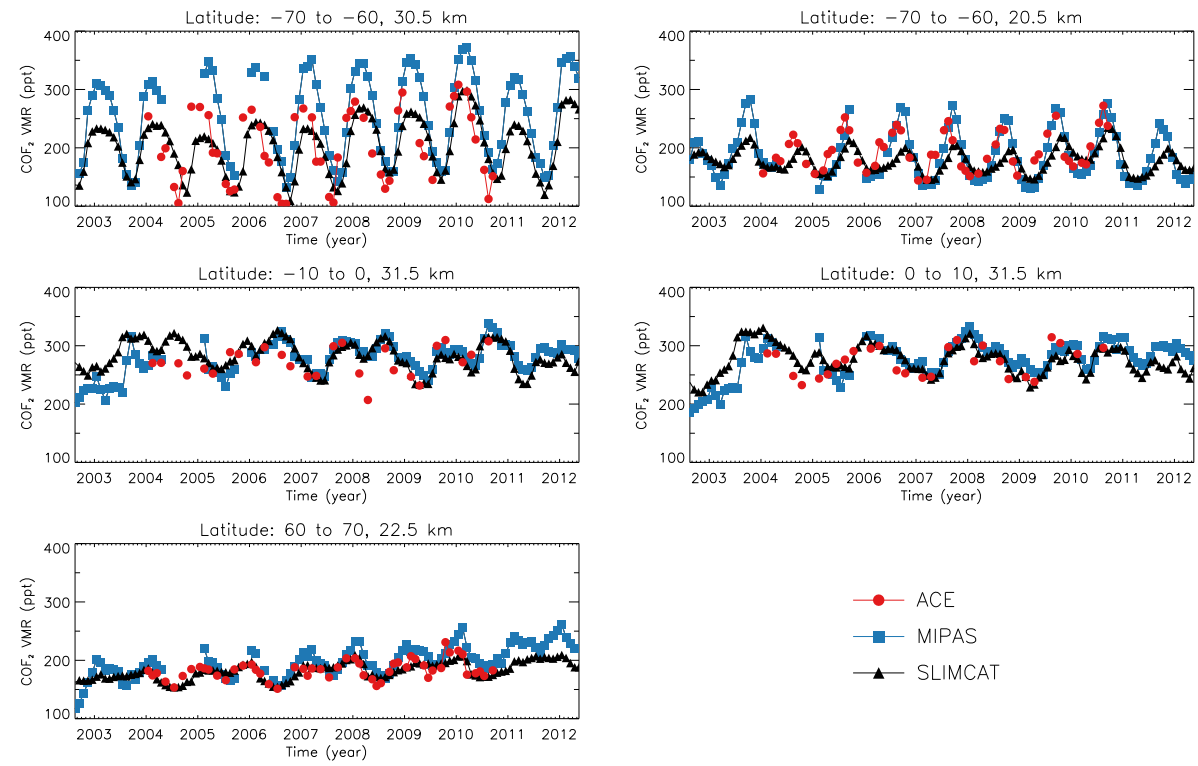

Figure 13. The ACE, MIPAS, and SLIMCAT COF 2 time series between July 2002 and April 2012 for five altitude-latitude bin combinations.

of all zonal means for each of the 12 months, $m$; and $\delta_{n m}$, although not used in its strict mathematical sense, is 1 when index $n$ corresponds to one of the months, $m$, and is 0 otherwise. In order to compare the three data sets, the same time period was used for each analysis. Such an approach essentially removes the annual cycle and the effect of biases in VMRs; the trend is simply equated to the "slope" of the linear regression between $\mathrm{C}^{z, \theta}(n)$ and the dependent variable $n / 12$. The inclusion of additional terms such as the annual cycle and its harmonics resulted in no additional improvement in the regression.

Figure 14 presents the annual percentage trends (January 2004 to September 2010) for ACE, MIPAS, and SLIM$\mathrm{CAT}$ as a function of latitude and altitude. The plotting range has been chosen to cover the maximum VMR features in the $\mathrm{COF}_{2}$ global distributions; this broadly follows the upperaltitude range of the actual ACE retrievals and removes portions of the MIPAS profiles that have the largest contributions from the a priori profile. Note that, whereas the MIPAS time series used to derive trends contains data for 67 distinct months in all latitude bands, the number of months of ACE data available varies from as low as 15 to as high as 63 in each latitude band. Errors were not explicitly treated in the linear regression of the SLIMCAT outputs, but they were for the MIPAS and ACE VMRs. Note that, as the MIPAS and ACE trends approach 0 , the ratio to their $1 \sigma$ uncertainties drops well below 1 . Broadly speaking, the trends for any ACE/MIPAS latitude-altitude region in Fig. 14 which appear predominantly blue or green become more statistically significant when the individual contributions are averaged.

The MIPAS plot in Fig. 14 indicates that, between 2004 and 2010, $\mathrm{COF}_{2}$ increased most rapidly (approaching $\sim 4 \%$ per year) at altitudes above $\sim 25 \mathrm{~km}$ in the southern latitudes and at altitudes below $\sim 25 \mathrm{~km}$ in the northern latitudes. The ACE plot broadly agrees with respect to these two regions of largest positive trend, although their magnitudes are slightly lower. Additionally, the ACE trends in the tropical region are predominantly negative, which somewhat agrees with SLIMCAT below $25 \mathrm{~km}$.

The SLIMCAT plot contains a number of features which agree with both the MIPAS and ACE plots. In particular, the SLIMCAT plot indicates a decrease in $\mathrm{COF}_{2}$ in the tropical region (between $20^{\circ} \mathrm{S}$ and $10^{\circ} \mathrm{N}$ ), although the largest decrease occurs at $\sim 27 \mathrm{~km}$ and $0^{\circ}$ latitude; ACE agrees better than MIPAS in this region, except for a narrow altitude range $\sim 30 \mathrm{~km}$ where the ACE trends are slightly positive. Outside the tropics, the SLIMCAT plot agrees better with MIPAS, in particular for the regions of largest positive trends, which occur at high southern latitudes above $30 \mathrm{~km}$ and northern latitudes below $\sim 25 \mathrm{~km}$.

An additional SLIMCAT run has been performed with dynamics arbitrarily fixed to those for the year 2000; results from this run give a "clean" $\mathrm{COF}_{2}$ signal without the complication of changes in stratospheric dynamics. Trends have been calculated in the same manner as above and plotted in the lowest panel of Fig. 14. Compared with trends for the "control" SLIMCAT run, those for the fixed-dynamics run lie predominantly between 0 and $1 \%$, with a relatively uniform distribution throughout the stratosphere. This indicates that the variations in SLIMCAT trends, and by extension the regions of agreement with MIPAS and ACE, result from changes in stratospheric dynamics between January 2004 and September 2010.

One might expect that the decreasing SLIMCAT trends over the 2004-2010 period in the lower tropical stratosphere, where the air is youngest, result directly from the decrease in 

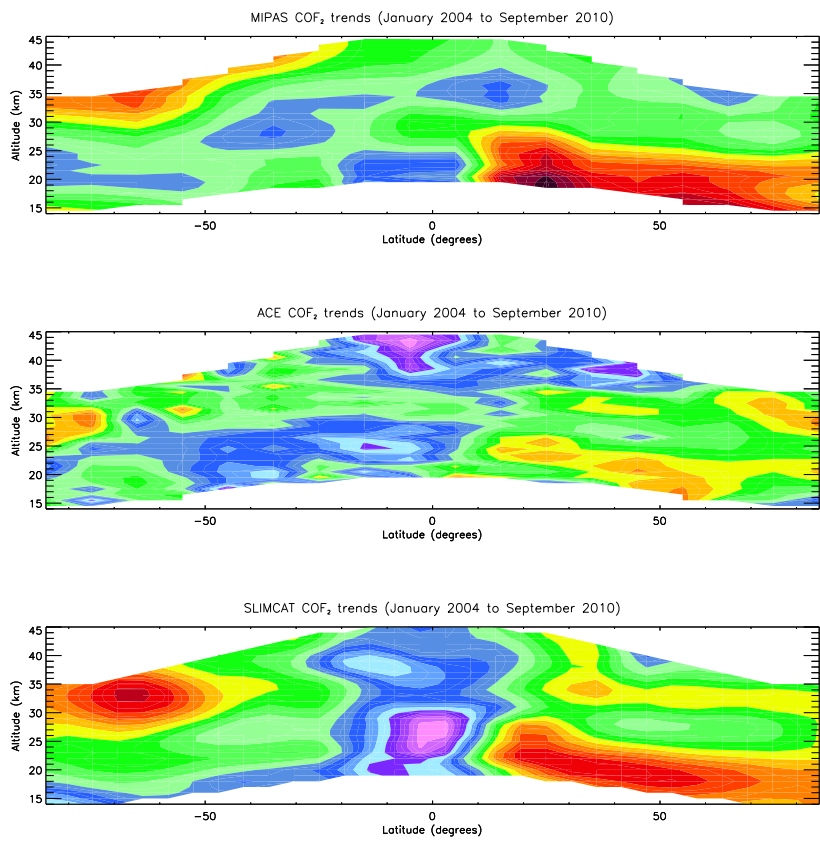

SLIMCAT COF, trends (January 2004 to September 2010) - fixed to 2000 dynamis
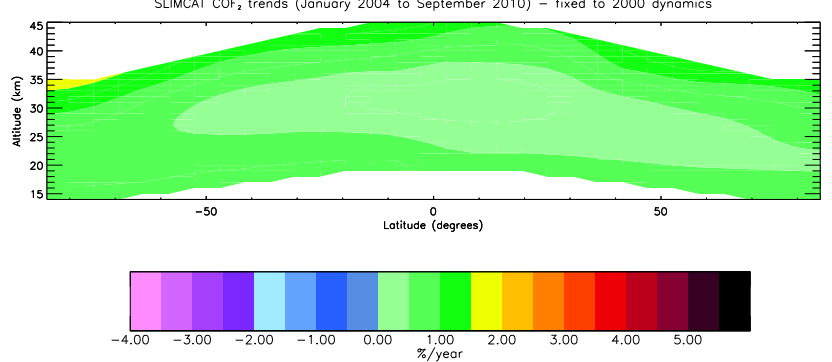

Figure 14. Annual percentage trends (January 2004 to September 2010) for ACE, MIPAS, and SLIMCAT as a function of latitude and altitude. A full discussion of these trends is provided in the text.

mean global surface mixing ratio of CFC-12 since $\sim 2003$ 2004 (WMO/UNEP, 2011); note that HCFC-22 produces $\mathrm{COF}_{2}$ at higher altitudes. However, the absence of any negative tropical trends in the fixed-dynamics SLIMCAT plot indicates that this feature must result from dynamical considerations.

The analyses used to force the SLIMCAT calculations provide information on the stratospheric circulation but do not allow for any rigorous explanation of the changing stratospheric dynamics that are responsible for the observed trends. Interestingly, the two regions of large positive trends in the ACE, MIPAS, and SLIMCAT plots correspond quite well to the regions of positive age of air trends, as reported by Stiller et al. (2012); see their Fig. 10. Additionally, the region of positive trends in the tropics $\sim 28-35 \mathrm{~km}$, contained in the ACE plot, more-or-less agrees with the corresponding feature in the age-of-air-trend plot. As discussed by Stiller et al. (2012), it is likely that variations in atmospheric mixing have occurred over the observation period. The regions of maximum $\mathrm{COF}_{2}$ trends must result from increased inmixing of $\mathrm{COF}_{2}$-rich air, possibly due to major sudden stratospheric mid-winter warmings. The negative trends in the tropics could result from an increase in the rate of upwelling over the observation period. MIPAS observations of CFC-11 and CFC-12, reported by Kellmann et al. (2012), reveal similar variations in trends over the globe. For example, despite these molecules slowly being removed from the atmosphere, a positive trend is readily observed in the stratosphere within $\sim 10-90^{\circ} \mathrm{S}$ and $\sim 22-30 \mathrm{~km}$ altitude.

Overall global trends in $\mathrm{COF}_{2}$ VMRs, weighted by the average VMRs at each altitude and latitude, have been calculated from the three data sets using errors in trends as determined from the linear regression: $0.30 \pm 0.44 \%$ year $^{-1}$ for ACE, $0.85 \pm 0.34 \%$ year $^{-1}$ for MIPAS, and $0.88 \%$ year $^{-1}$ for SLIMCAT. Note that these values only apply to the January 2004 to September 2010 time period. Any spectroscopic deficiencies that might lead to regional biases in the ACE and MIPAS data sets should have been removed by taking percentage anomalies; however there still remains the possibility of systematic errors that contribute to time-dependent biases. The pressure-temperature retrievals for ACE v3.0 processing assume a rate of increase of $1.5 \mathrm{ppm}$ year $^{-1}$ for the $\mathrm{CO}_{2}$ VMRs, which are assumed to have a single profile shape for all locations and seasons. This rate of increase is lower than the accepted value of $1.90-1.95 \mathrm{ppm} y e a r^{-1}\left(0.5 \%\right.$ year $\left.^{-1}\right)$ as used, for example, in IG2 $\mathrm{CO}_{2}$ profiles for MIPAS retrievals. By the end of the time series, ACE v3.0 $\mathrm{CO}_{2}$ VMRs are too low by $\sim 0.7 \%$. This translates into a small timedependent negative bias in $\mathrm{COF}_{2} \mathrm{VMR}$, meaning that the trend derived from ACE v3.0 data is biased low by on average $\sim 0.1 \%$ year $^{-1}$, although it is not obvious how the bias varies with latitude and altitude.

Plans are currently underway to create a new ACE processing version 4.0, in which it is assumed that the $\mathrm{CO}_{2} \mathrm{VMR}$ increases by $0.5 \%$ year $^{-1}$ and in which age of air considerations are used to generate the vertical $\mathrm{CO}_{2}$ VMR profile as a function of latitude and time of year (G. C. Toon, personal communication, 2012). It is anticipated that the new v4.0 will enable more accurate trends to be derived. The ACE-FTS continues to take atmospheric measurements from orbit, with only minor loss in performance; it will be possible to extend the $\mathrm{COF}_{2}$ time series to the present day and beyond.

\section{Conclusions}

$\mathrm{COF}_{2}$ is the second-most abundant inorganic fluorine reservoir in the stratosphere, with main sources being the atmospheric degradation of CFC-12 $\left(\mathrm{CCl}_{2} \mathrm{~F}_{2}\right)$, HCFC-22 $\left(\mathrm{CHF}_{2} \mathrm{Cl}\right)$, and $\mathrm{CFC}-113\left(\mathrm{CF}_{2} \mathrm{ClCFCl}_{2}\right)$, species whose emissions are predominantly anthropogenic.

This work reports the first global distributions of carbonyl fluoride in the Earth's atmosphere using infrared satellite remote-sensing measurements by the ACE-FTS, which has 
been recording atmospheric spectra since 2004, and the MIPAS instrument, which has recorded thermal emission atmospheric spectra between 2002 and 2012. The observations reveal a high degree of seasonal and latitudinal variability over the course of a year, and agree well with the output of the SLIMCAT model, although MIPAS VMRs are biased high relative to ACE by as much as $\sim 30 \%$. This MIPAS-ACE bias is believed to arise predominantly from the large $\mathrm{COF}_{2}$ spectroscopic errors, which make differing contributions to the ACE and MIPAS profiles due to the different microwindows used in the two retrieval schemes.

The maximum in the $\mathrm{COF}_{2} \mathrm{VMR}$ distribution occurs at $\sim 30-35 \mathrm{~km}$ altitude in the tropics, where solar insolation is highest; this region is dominated by $\mathrm{COF}_{2}$ formed from the photolysis of CFC-12 and CFC-113. The first-order loss rates of the main $\mathrm{COF}_{2}$ precursors are symmetrical between the hemispheres, except for the $\mathrm{HCFC}-22+\mathrm{OH}$ reaction, which is temperature-dependent; a secondary maximum at $\sim 25-30 \mathrm{~km}$ altitude is present at high latitudes in SH summer due to the mid-stratosphere being around $10 \mathrm{~K}$ warmer than the corresponding location in the $\mathrm{NH}$ summer. There is also asymmetry in the distribution of $\mathrm{COF}_{2}$ precursors due to differences in the meridional Brewer-Dobson circulation, with stronger mixing to the pole in the north and stronger descent in the south; this results in larger VMRs at mid- and high latitudes in the $\mathrm{SH}$.

Between January 2004 and September $2010 \mathrm{COF}_{2}$ grew most rapidly at altitudes above $\sim 25 \mathrm{~km}$ in the southern latitudes and at altitudes below $\sim 25 \mathrm{~km}$ in the northern latitudes, whereas it declined most rapidly in the tropics. These variations are attributed to changes in stratospheric dynamics over the observation period. The overall $\mathrm{COF}_{2}$ global trend over this period is calculated as $0.85 \pm 0.34$ (MIPAS), $0.30 \pm 0.44$ (ACE), and $0.88 \%$ year $^{-1}$ (SLIMCAT).

Author contributions. Based on an idea from P. F. Bernath, J. J. Harrison devised the study and performed the data analysis. A. Dudhia performed the MIPAS retrievals, and S. Cai filtered and prepared the data for analysis. C. D. Boone performed the ACEFTS retrievals, and J. J. Harrison filtered and prepared the data for analysis. P. F. Bernath allowed the use of ACE data in this work. M. P. Chipperfield and S. Dhomse ran the SLIMCAT model and provided additional explanation of the outputs. J. J. Harrison prepared the manuscript with contributions from M. P. Chipperfield and A. Dudhia.

Acknowledgements. The authors wish to thank the Natural Environment Research Council (NERC) for supporting J. J. Harrison through grant NE/I022663/1. The ACE satellite mission is funded primarily by the Canadian Space Agency (CSA). The SLIMCAT modelling work at Leeds is supported by NERC NCAS (National Centre for Atmospheric Science) and NCEO (National Centre for Earth Observation). We thank Wuhu Feng (NCAS) for help with the model.

Edited by: T. von Clarmann

\section{References}

Bernath, P. F., McElroy, C. T., Abrams, M. C., Boone, C. D., Butler, M., Camy-Peyret, C., Carleer, M., Clerbaux, C., Coheur, P.-F., Colin, R., DeCola, P., DeMazière, M., Drummond, J. R., Dufour, D., Evans, W. F. J., Fast, H., Fussen, D., Gilbert, K., Jennings, D. E., Llewellyn, E. J., Lowe, R. P., Mahieu, E., McConnell, J. C., McHugh, M., McLeod, S. D., Michaud, R., Midwinter, C., Nassar, R., Nichitiu, F., Nowlan, C., Rinsland, C. P., Rochon, Y. J., Rowlands, N., Semeniuk, K., Simon, P., Skelton, R., Sloan, J. J., Soucy, M.-A., Strong, K., Tremblay, P., Turnbull, D., Walker, K. A., Walkty, I., Wardle, D. A., Wehrle, V., Zander, R., and Zou, J.: Atmospheric Chemistry Experiment (ACE): Mission overview, Geophys. Res. Lett., 32, L15S01, doi:10.1029/2005GL022386, 2005.

Boone, C. D., Nassar, R., Walker, K. A., Rochon, Y., McLeod, S. D., Rinsland, C. P., and Bernath, P. F.: Retrievals for the Atmospheric Chemistry Experiment Fourier Transform Spectrometer, Appl. Optics, 44, 7218-7231, 2005.

Boone, C. D., Walker, K. A., and Bernath, P. F.: Version 3 retrievals for the Atmospheric Chemistry Experiment Fourier Transform Spectrometer (ACE-FTS), in: The Atmospheric Chemistry Experiment ACE at 10: a Solar Occultation Anthology, edited by: Bernath, P. F., A. Deepak Publishing, Hampton, Virginia, USA, 103-127, available at: http://acebox2.uwaterloo.ca/publications/ 2013/Version3.5retreivals2013.pdf (last access: 1 July 2014), 2013.

Brown, A. T., Chipperfield, M. P., Boone, C., Wilson, C., Walker, K. A., and Bernath, P. F.: Trends in atmospheric halogen containing gases since 2004, J. Quant. Spectrosc. Ra., 112, 2552-2566, 2011.

Brown, A. T., Chipperfield, M. P., Richards, N. A. D., Boone, C., and Bernath, P. F.: Global stratospheric fluorine inventory for 2004-2009 from Atmospheric Chemistry Experiment Fourier Transform Spectrometer (ACE-FTS) measurements and SLIMCAT model simulations, Atmos. Chem. Phys., 14, 267-282, doi:10.5194/acp-14-267-2014, 2014.

Chipperfield, M. P.: Multiannual simulations with a threedimensional chemical transport model, J. Geophys. Res., 104, 1781-1805, doi:10.1029/98jd02597, 1999.

Chipperfield, M. P.: New version of the TOMCAT/SLIMCAT offline chemical transport model: Intercomparison of stratospheric tracer experiments, Q. J. Roy. Meteorol. Soc., 132, 1179-1203, 2006.

Clerbaux, C., Coheur, P.-F., Hurtmans, D., Barret, B., Carleer, M., Colin, R., Semeniuk, K., McConnell, J. C., Boone, C., and Bernath, P.:, Carbon monoxide distribution from the ACEFTS solar occultation measurements, Geophys. Res. Lett., 32, L16S01, doi:10.1029/2005GL022394, 2005.

Dhomse, S., Chipperfield, M. P., Feng, W., and Haigh, J. D.: Solar response in tropical stratospheric ozone: a 3-D chemical transport model study using ERA reanalyses, Atmos. Chem. Phys., 11, 12773-12786, doi:10.5194/acp-11-12773-2011, 2011.

Duchatelet, P., Mahieu, E., Ruhnke, R., Feng, W., Chipperfield, M., Demoulin, P., Bernath, P., Boone, C. D., Walker, K. A., Servais, C., and Flock, O.: An approach to retrieve information on the carbonyl fluoride $\left(\mathrm{COF}_{2}\right)$ vertical distributions above Jungfraujoch by FTIR multi-spectrum multi-window fitting, Atmos. Chem. Phys., 9, 9027-9042, doi:10.5194/acp-9-9027-2009, 2009. 
Duchatelet, P., Demoulin, P., Hase, F., Ruhnke, R., Feng, W., Chipperfield, M. P., Bernath, P. F., Boone, C. D., Walker, K. A., and Mahieu, E.: Hydrogen fluoride total and partial column time series above the Jungfraujoch from long-term FTIR measurements: Impact of the line-shape model, characterization of the error budget and seasonal cycle, and comparison with satellite and model data, J. Geophys. Res., 115, D22306, doi:10.1029/2010JD014677, 2010.

Feng, W., Chipperfield, M. P., Dorf, M., Pfeilsticker, K., and Ricaud, P.: Mid-latitude ozone changes: studies with a 3-D CTM forced by ERA-40 analyses, Atmos. Chem. Phys., 7, 2357-2369, doi:10.5194/acp-7-2357-2007, 2007.

Fischer, H., Birk, M., Blom, C., Carli, B., Carlotti, M., von Clarmann, T., Delbouille, L., Dudhia, A., Ehhalt, D., Endemann, M., Flaud, J. M., Gessner, R., Kleinert, A., Koopman, R., Langen, J., López-Puertas, M., Mosner, P., Nett, H., Oelhaf, H., Perron, G., Remedios, J., Ridolfi, M., Stiller, G., and Zander, R.: MIPAS: an instrument for atmospheric and climate research, Atmos. Chem. Phys., 8, 2151-2188, doi:10.5194/acp-8-2151-2008, 2008.

Flaud, J.-M., Brizzi, G., Carlotti, M., Perrin, A., and Ridolfi, M.: MIPAS database: Validation of $\mathrm{HNO}_{3}$ line parameters using MIPAS satellite measurements, Atmos. Chem. Phys., 6, 5037-5048, doi:10.5194/acp-6-5037-2006, 2006.

Gribble, G. W.: Naturally Occurring Organofluorines, in: Handbook of Environmental Chemistry, Vol. 3, Part N: Organofluorines, edited by: Neilson, A. H., Springer-Verlag: Berlin Heidelberg, 2002.

Harrison, J. J. and Bernath, P. F.: ACE-FTS observations of acetonitrile in the lower stratosphere, Atmos. Chem. Phys., 13, 74057413, doi:10.5194/acp-13-7405-2013, 2013.

Hartmann, J. M., Boulet, C., and Robert, D.: Collisional Effects on Molecular Spectra, Laboratory experiments and models, consequences for applications, Elsevier: Oxford, 2008.

Irion, F. W., Gunson, M. R., Toon, G. C., Chang, A. Y., Eldering, A., Mahieu, E., Manney, G. L., Michelsen, H. A., Moyer, E. J., Newchurch, M. J., Osterman, G. B., Rinsland, C. P., Salawitch, R. J., Sen, B., Yung, Y. L., and Zander, R.: Atmospheric Trace Molecule Spectroscopy (ATMOS) Experiment Version 3 data retrievals, Appl. Optics, 41, 6968-6979, 2002.

Kellmann, S., von Clarmann, T., Stiller, G. P., Eckert, E., Glatthor, N., Höpfner, M., Kiefer, M., Orphal, J., Funke, B., Grabowski, U., Linden, A., Dutton, G. S., and Elkins, J. W.: Global CFC-11 $\left(\mathrm{CCl}_{3} \mathrm{~F}\right)$ and $\mathrm{CFC}-12\left(\mathrm{CCl}_{2} \mathrm{~F}_{2}\right)$ measurements with the Michelson Interferometer for Passive Atmospheric Sounding (MIPAS): retrieval, climatologies and trends, Atmos. Chem. Phys., 12, 11857-11875, doi:10.5194/acp-12-11857-2012, 2012.

May, R. D.: Line intensities and collisional-broadening parameters for the $\mathrm{v}_{4}$ and $\mathrm{v}_{6}$ bands of carbonyl fluoride, J. Quant. Spectrosc. Ra., 48, 701-712, 1992.

Mélen, F., Mahieu, E., Zander, R., Rinsland, C. P., Demoulin, P., Roland, G., Delbouille, L., and Servais, C.: Vertical column abundances of $\mathrm{COF}_{2}$ above the Jungfraujoch Station, derived from ground-based infrared solar observations, J. Atmos. Chem., 29, 119-134, 1998.

Monge-Sanz, B. M., Chipperfield, M. P., Simmons, A. J., and Uppala, S. M.: Mean age of air and transport in a CTM: Comparison of different ECMWF analyses, Geophys. Res. Lett., 34, L04801, doi:10.1029/2006g1028515, 2007.
Plumb, R. A. and Ko, M. K. W.: Interrelationships between mixing ratios of long-lived stratospheric constituents, J. Geophys. Res., 97, 10145-10156, 1992.

Remedios, J. J., Leigh, R. J., Waterfall, A. M., Moore, D. P., Sembhi, H., Parkes, I., Greenhough, J., Chipperfield, M. P., and Hauglustaine, D.: MIPAS reference atmospheres and comparisons to V4.61/V4.62 MIPAS level 2 geophysical data sets, Atmos. Chem. Phys. Discuss., 7, 9973-10017, doi:10.5194/acpd-79973-2007, 2007.

Ricaud, P. and Lefevre, F.: Fluorine in the atmosphere, in: Fluorine and the Environment: Atmospheric Chemistry, Emissions, \& Lithosphere, edited by: Tressaud, A., Elsevier, Oxford, UK, 2006.

Rinsland, C. P., Zander, R., Brown, L. R., Farmer, C. B., Park, J. H., Norton, R. H., Russell III, J. M., and Raper, O. F.: Detection of carbonyl fluoride in the stratosphere, Geophys. Res. Lett., 13, 769-772, 1986.

Rodgers, C. D.: Inverse methods for atmospheric sounding: Theory and Practice, Volume 2 of Series on Atmospheric, Oceanic and Planetary Physics, World Scientific Co. Pte. Ltd., Singapore, 2000.

Rothman, L., Jacquemart, D., Barbe, A., Benner, C. D., Birk, M., Brown, L. R., Carleer, M. R., Chackerian Jr., C., Chance, K., Coudert, L. H., Dana, V., Devi, V. M., Flaud, J.-M., Gamache, R. R., Goldman, A., Hartmann, J.-M., Jucks, J. W., Maki, A. G., Mandin, J.-Y., Massie, S. T., Orphal, J., Perrin, A., Rinsland, C. P., Smith, M., Tennyson, J., Tolchenov, R. N., Toth, R. A., Vander Auwera, J., Varanasi, P., and Wagner, G.: The HITRAN 2004 molecular spectroscopic database, J. Quant. Spectrosc. Ra., 96, 193-204, 2005.

Sharpe, S. W., Johnson, T. J., Sams, R. L., Chu, P. M., Rhoderick, G. C., and Johnson, P. A.: Gas-phase databases for quantitative infrared spectroscopy, Appl Spectrosc., 58, 1452-1461, 2004.

Spang, R., Remedios, J. J., and Barkley, M.: Colour indices for the detection and differentiation of cloud types in infra-red limb emission spectra, Adv. Space Res., 33, 1041-1047, 2004.

Stiller, G. P., von Clarmann, T., Haenel, F., Funke, B., Glatthor, N., Grabowski, U., Kellmann, S., Kiefer, M., Linden, A., Lossow, S., and López-Puertas, M.: Observed temporal evolution of global mean age of stratospheric air for the 2002 to 2010 period, Atmos. Chem. Phys., 12, 3311-3331, doi:10.5194/acp-12-33112012, 2012.

Velazco, V. A., Toon, G. C., Blavier, J.-F. L., Kleinböhl, A., Manney, G. L., Daffer, W. H., Bernath, P. F., Walker, K. A., and Boone, C.: Validation of the Atmospheric Chemistry Experiment by noncoincident MkIV balloon profiles, J. Geophys. Res., 116, D06306, doi:10.1029/2010jd014928, 2011.

von Clarmann, T.: Smoothing error pitfalls, Atmos. Meas. Tech., 7, 3023-3034, doi:10.5194/amt-7-3023-2014, 2014.

WMO/UNEP: Scientific Assessment of Ozone Depletion: 2010, Global Ozone Research and Monitoring Project-Report No. 52, 516 pp., Geneva, Switzerland, 2011.

Zander, R., Rinsland, C. P., Mahieu, E., Gunson, M. R., Farmer, C. B., Abrams, M. C., and Ko, M. K. W.: Increase of carbonyl fluoride $\left(\mathrm{COF}_{2}\right)$ in the stratosphere and its contribution to the 1992 budget of inorganic fluorine in the upper stratosphere, J. Geophys. Res., 99, 16737-16743, 1994. 